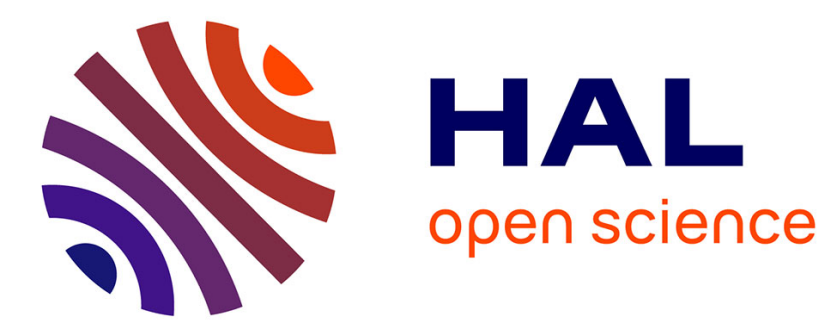

\title{
A Multi-Agent Advanced Traveler Information System for Optimal Trip Planning in a Co-Modal Framework
}

Mariagrazia Dotoli, Hayfa Zgaya, Carmine Russo, Slim Hammadi

\section{To cite this version:}

Mariagrazia Dotoli, Hayfa Zgaya, Carmine Russo, Slim Hammadi. A Multi-Agent Advanced Traveler Information System for Optimal Trip Planning in a Co-Modal Framework. IEEE Transactions on Intelligent Transportation Systems, 2017, 18 (9), pp.2397 - 2412. 10.1109/TITS.2016.2645278 . hal01713616v1

\section{HAL Id: hal-01713616 https://hal.science/hal-01713616v1}

Submitted on 20 Feb 2018 (v1), last revised 2 Mar 2018 (v2)

HAL is a multi-disciplinary open access archive for the deposit and dissemination of scientific research documents, whether they are published or not. The documents may come from teaching and research institutions in France or abroad, or from public or private research centers.
L'archive ouverte pluridisciplinaire HAL, est destinée au dépôt et à la diffusion de documents scientifiques de niveau recherche, publiés ou non, émanant des établissements d'enseignement et de recherche français ou étrangers, des laboratoires publics ou privés. 


\title{
A Multi-Agent Advanced Traveler Information System for Optimal Trip Planning in a Co-Modal Framework
}

\author{
Mariagrazia Dotoli, Senior Member, IEEE, Hayfa Zgaya, Carmine Russo, \\ and Slim Hammadi, Senior Member, IEEE
}

\begin{abstract}
We present an advanced traveler information system (ATIS) for public and private transportation, including vehicle sharing and pooling services. The ATIS uses an agentbased architecture and multi-objective optimization to answer trip planning requests from multiple users in a co-modal setting, considering vehicle preferences and conflicting criteria. At each set of users' requests, the transportation network is represented by a co-modal graph that allows decomposing the trip planning problem into smaller tasks: the shortest routes between the network nodes are determined and then combined to obtain possible itineraries. Using multi-objective optimization, the set of uservehicle-route combinations according to the users' preferences is determined, ranking all possible route agents' coalitions. The ATIS is tested for the real case study of the Lille metropolitan area (Nord Pas de Calais, France).
\end{abstract}

Index Terms-Advanced traveler information system, trip planning, public transport, private transport, co-modal transport, multi-agent systems, directed graphs, optimization.

\section{INTRODUCTION}

\section{A. Contribution}

$\mathbf{S}$ HARED

transportation services are emerging [5]. In multi-modal transportation users employ at least two different types of means of transport. Co-modality, instead, arises from the need to convey people on a single means of transport to reduce the impact on environment, costs, and accidents. Hence, co-modality refers to the optimal use of different transportation modes on their own or in combination, taking advantage of ridesharing (the sharing of vehicles by passengers). Information and communication technologies may support the development of advanced tools for passengers allowing the effective integration of transportation modalities [11], [25]. As a result,

Manuscript received October 14, 2015; revised March 23, 2016 and August 31, 2016; accepted December 18, 2016. Date of publication January 23, 2017; date of current version August 28, 2017. The Associate Editor for this paper was D. Chen.

M. Dotoli is with the Department of Electrical and Information Engineering, Politecnico di Bari, 70125 Bari, Italy (e-mail: mariagrazia.dotoli@poliba.it).

$\mathrm{H}$. Zgaya is with the ILIS-Lille Institute of Healthcare Engineering, Université de Lille 2, 59000 Lille, France (e-mail: hayfa.zgayabiau@univlille2.fr).

C. Russo is with the Magneti Marelli-Powertrain Research, 10135 Torino, Italy (e-mail: cr88fg@gmail.com).

S. Hammadi is with the CRISTAL-Research Centre in Computer Science, Signals and Automatic Systems of Lille, École Centrale de Lille, 59651 Lille, France (e-mail: slim.hammadi@ec-lille.fr).

Digital Object Identifier 10.1109/TITS.2016.2645278 the field of intelligent transportation systems and particularly of Advanced Traveler Information Systems (ATISs) is rapidly growing [44]. An ATIS may be defined as a system providing pre-trip and real time information on departures, routes, and modes of travel. However, the related literature in the field of passengers' co-modal transportation services is scarce, showing a need for ATISs supporting sustainability-oriented decisions.

This paper aims at filling this gap by a multi-agent ATIS for passengers' pre-trip planning considering co-modal itineraries with multiple preference criteria, taking into account public and private transportation, and including vehicle sharing and pooling. Users request itineraries to the ATIS, with given (eventually different) origin and destination pairs and arrival/departure time windows, specifying their preferences by an ordered sequence of criteria. The ATIS matches requests with information in transportation operators' databases and chooses transportation means and routes. It provides the routes answering requests and optimizing travel time, travel cost, and gas emissions. To the best of the authors' knowledge, no ATIS for trip planning exists in the literature for trip planning both with private and public transport in a co-modal and multi-objective framework, i.e., with multiple users and preferences. Moreover, with respect to the previous works by the authors, we remark here that the paper enhances and extends three previous contributions [12], [20], [21]. The ATIS architecture borrows the multi-agent systems paradigm from [20], improving the vehicle/operator/route/users association, which is here determined in a stand-alone way without using an external software as in [2] but rather representing, as in [21], the transportation network by a co-modal graph. With respect to [21], however, here two improvements are provided. First, we enhance the trip calculation defining route agents to represent the possible routes composing the itinerary solution path and employing an agent coalition mechanism to determine the best person-to-vehicle assignment for the concerned route using genetic optimization. Second, the user can express his preference among different transportation means and define a descending order of priority of multiple criteria (cost, time, and emissions in the case study). Finally, we remark that the paper is a deeply revised version of [12]. Here we detail the multi-agent architecture, only sketched in [12], by describing all the agents operations. Moreover, we enhance the case study presenting a totally new 
urban scenario together with an extra-urban scenario revisited from [12].

\section{B. Related Works and Paper Positioning}

Summaries of research contributions on passengers pretrip route advisory systems are in [8]-[10], [44], and [45]. Numerous projects were devoted to systems that recommend travelers a combination of transportation means for door to door journeys [6], [13], [16]-[19], [23], [24], [29], [32], [37], [39], [44]. However, all these works neither consider shared public nor pooled private vehicles. Moreover, they solve the pre-trip planning for a single user, disregarding sustainability.

An interesting work is proposed by Zhang et al. [45] who present an ATIS for multimodal transport considering both private and public transportation, considering time, cost, effort and comfort attributes. However, the approach is devoted to single user trip planning, i.e., it does not take into account the co-modal approach. Moreover, private and public networks are modeled separately and integrated only as a last step, determining the trip by a centralized approach, leading to long computational times. Other works use artificial intelligence approaches to develop an ATIS capable of some adaptability. For instance, Park et al. [30] present an adaptive route choice system using a rule-based decision-tree approach to incorporate an adaptation process into a user's route selection rule. However, this work does not take into account multimodality. Further, Arentze [3] presents an ATIS for trip planning on multi-modal transport networks using Bayesian inference to adaptively learn the user's preference. However, this approach is devoted to answering single users' requests. More recently, several advanced public transportation information systems have been developed, determining the shortest itineraries in terms of travel time or cost through online web-based applications (see the review in [46]). However, in all the recalled ATISs the problem is solved by a classical centralized approach and without any co-modality objective. In fact, the discussed works typically consider only a user request at a time. On the contrary, in our approach the ATIS optimizes itineraries considering all requests at the same time, taking into account the co-modal context, so that the ATIS is intrinsically devoted to enhancing sustainable mobility. Further, thanks to the multi-agent systems framework, we are able to consider each mode of transportation and each provider of the transportation network in a distributed way, solving the problem locally and combining solutions into an optimal set of global solutions, so that we deeply reduce the computational complexity.

In the context of multi-agent approaches [7] for pre-trip planning, in [33] a personal intelligent traveler assistant for train passengers is developed. Using agents to communicate via PDAs and a wireless network, the ATIS provides the user with a personalized route advice taking into account his preferences. In addition, Yin and Griss [42] present an agentbased multi-modal traveler information system that fuses information from multiple sources on routes, congestion, incidents, weather, alternative transit modes and schedules, resulting in a user-friendly personalized assistant to a single traveler.
However, both [33] and [42] present ATISs for single users. Further, in [35] a multi-agent system is presented addressing a set of requests in a temporal window by a vehicle routing with a time window approach. A tree is built, with the pieces of the overall path, and the solution is found through a research into this tree. However, the resulting complexity of the algorithm in [35] is exponential with the number of leaves of the tree. Further, in [47] a multi-agent route planner performs a local optimization by each agent with respect to different criteria: traffic load, road safety, road type, and weather condition. After the local optimization, a global graph is built and an agent determines the travelling time. Differently from [35] and [47], in our multi-agent ATIS each agent determines a piece of the global solution, so that with the agents' coalition, an overall solution may be found with a reduced complexity with respect to the centralized case. Moreover, Adler et al. [2] propose a multi-agent approach for cooperative traffic management that employs negotiation to solve the pre-trip route assignment in a multi-objective framework, but the system does not take into account sustainability that is essential in a co-modal framework.

Summing up, the cited studies on ATISs typically refer to public transportation services, neither taking into account vehicle sharing services nor vehicle pooling ones. Moreover, previous works typically consider one user, only one transportation service and use centralized information. On the contrary, the ATIS here presented is able to consider any type of public/private transportation services and operators, including vehicle sharing/pooling services and operators.

\section{The Co-Modal Trip Planning Problem}

\section{A. The Problem Presentation}

The scope of the proposed ATIS is to satisfy users' requests for itineraries by answering trip requests respecting preferences. To this aim, the ATIS employs the notion of co-modality: combining all possible means of public transport with private transportations services, i.e., using different modes on their own or in combination to reach an effective and sustainable utilization of resources. To improve sustainability, the ATIS considers for each transportation service multiple operators that may offer the same service.

We assume that multiple users formulate simultaneously (or in a short time window) a set of requests. Hence, the ATIS determines feasible decompositions of each itinerary or route, i.e., subroutes, by recognizing similarities in order to associate different users to the same vehicle and transportation service (so as to satisfy the co-modality requirements). For a given route or subroute, several transportation possibilities may exist with different vehicles, which may all be available for that route through the same time window. The problem is thus to choose the most effective route combination for a given user, taking into account his constraints and preferences in terms of preferred means of transport and conflicting criteria, while considering the co-modality requirements. We adopt an aggregative approach to obtain the most effective solution by a compromise between criteria (transport time, total cost, and total gas emissions). By means of a weighted sum function, the 
aggregation method reduces the number of conflicting criteria by judiciously choosing the weights combining them into a single optimization criterion so that two conflicting criteria cannot be improved or deteriorated at the same time.

We remark that in co-modal transport systems the optimization criteria are typically multiple and conflicting. They depend on the physical situation (geolocalization of transport means), on the topological configuration of the transportation network, as well as on technical capabilities, institutional capacities, financing support, and political decisions. In addition, passengers' preferences usually imply a conflicting situation in order to reach low cost, high quality services, rapid itineraries, comfort, safety and security. Here we choose three classical criteria that are clearly conflicting: travel time, travel cost, and gas emissions. For example, in the carpooling mode, the driver can make a detour to pick up new passengers. This detour increases the transport time and decreases the transport cost. In fact, the total cost of the itinerary is cheaper for each passenger because the total costs involved are shared. Exactly because of the complicated nature of the problem, and due to the presence of conflicting criteria, we propose a multi-criteria decision making ATIS.

\section{B. The Mathematical Formulation}

The co-modal transportation problem is complex because each transport service has several operators and each transport operator may offer several means of transport, knowing that the same means of transport may be offered by several different operators. Hence, at the time of the users' requests, our problem may be defined as follows. The ATIS inputs are:

- a set of $n$ available transportation services $\boldsymbol{T S}=\left\{T S_{1}, \ldots, T S_{i}, \ldots, T S_{n}\right\}$ (e.g., public transport, public vehicle sharing, private vehicle pooling, etc.);

- a number $o_{i}$ of transportation operators $T O_{i 1}, \ldots, T O_{i j}, \ldots, T O_{i o_{i}}$ for each $i$-th service with $i \in\{1, \ldots, n\}$;

- a number $q_{i j}$ of available transportation means $T M_{i j 1}, \ldots, T M_{i j y}, \ldots, T M_{i j q_{i j}} \in \boldsymbol{T M}$ for each $j$ th operator of the $i$-th service, where $\boldsymbol{T M}$ is the set of all transportation means (bus, train, subway, car, etc.);

- a set $\boldsymbol{C}=\left\{c_{1}, \ldots, c_{\gamma}, \ldots, c_{c}\right\}$ of c criteria according to which the optimal and co-modal trip planning is to be determined;

- a set $\boldsymbol{I}=\left\{i_{1}, \ldots, i_{k}, \ldots, i_{K}\right\}$ of $K$ itinerary requests each represented by a five-tuple $i_{k}=\left(d_{k}, a_{k}, W_{k}, V_{k}\right.$, $\left.C_{k}\right) \in I$ corresponding to the $k$-th user with $k=1, \ldots, K$, from a departure point $d_{k}$ to an arrival point $a_{k}$ and through a time window $W_{k}=\left(t_{d k}, t_{a k}\right)$ where $t_{d k}\left(t_{a k}\right)$ is the minimum (maximum) allowed departure (arrival) time by the user from $d_{k}$ (to $a_{k}$ ), with $V_{k} \in \boldsymbol{T} \boldsymbol{M}$ the list of transportation means allowed by the user and $C_{k}$ the ordered list of the c criteria in a descending order of importance for the user.

Given these inputs, the ATIS has to determine in a short time (i.e., before the next set of requests), the outputs:

- the set $S=\left\{S_{1}, \ldots, S_{k}, \ldots, S_{K}\right\}$ of route solutions where the generic itinerary is such that $S_{k} \in \boldsymbol{R} \boldsymbol{C}_{\mathbf{k}}$ with $\boldsymbol{R} \boldsymbol{C}_{\mathbf{k}}$

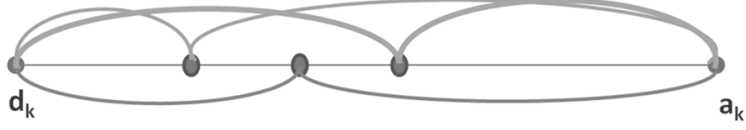

Fig. 1. Example of set of route solutions $\boldsymbol{R} \boldsymbol{C}_{\mathbf{k}}$ for request $i_{k}$.

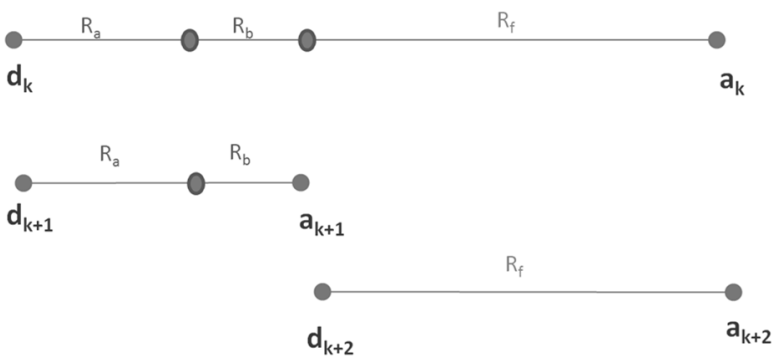

Fig. 2. Example of a set of users' requests sharing the same subroutes.

the set of all the possible $P_{k}=\operatorname{Card}\left(\boldsymbol{R} \boldsymbol{C}_{\mathbf{k}}\right)$ routes that may answer the $k$-th user's request $i_{k}$; Figure 1 shows an example of a set of route solutions $\boldsymbol{R} \boldsymbol{C}_{\mathbf{k}}$ for a request $i_{k}$. Each solution is represented by a different color. As a result, for this example there are four possible itineraries generated by the ATIS for user $k$, i.e., $P_{k}=\operatorname{Card}\left(\boldsymbol{R} \boldsymbol{C}_{\mathbf{k}}\right)=4$;

- for each request $k=1, \ldots, K$ the set $\boldsymbol{R} \boldsymbol{C}_{\mathrm{k}}=\left\{\mathrm{RC}_{\mathrm{k}, \mathrm{p}}\right.$, $\left.p \in\left\{1 \ldots P_{k}\right\}\right\} \mathrm{RC}_{\mathrm{k}}=\left\{\mathrm{RC}_{\mathrm{k}, \mathrm{p}}, \mathrm{p} \in[1 \ldots \mathrm{P}]\right\}$ of all the $P_{k}=$ Card $\left(\boldsymbol{R} \boldsymbol{C}_{\mathbf{k}}\right)$ possible route combinations $R C_{k, p}$ that can serve the request $i_{k}$ by combining a succession of subroutes into complete routes from the origin $d_{k}$ to destination $a_{k}$, where $R C_{k, p}$ is defined as a succession of subroutes $R_{g}$ with $g \in\{1, \ldots, X\}$, with $X$ the total number of all possible subroutes concerned by all users' requests at the considered time and $T_{k}$ the number of different subroutes, i.e., of different transportation means, that can serve in total the $k$-th user. Figure 2 shows an example with $X=3$ subroutes for $K=3$ requests with $T_{1}=3, T_{2}=2, T_{3}=1$. Moreover, Fig. 3 presents an example of route combinations for a request $i_{k}$, where it holds: $X=9, P_{k}=4$, and the 4 alternative route combinations each employ respectively 2, 4, 2, 2 subroutes of the 9 available ones;

- the set $\Lambda=\left\{\left(u_{1}, V_{1}\right), \ldots,\left(u_{g}, V_{g}\right), \ldots,\left(u_{G}, V_{G}\right)\right\}$ of ordered couples containing all lists $u_{g}$ of requests sharing the departure point $d_{g}$ and arrival point $a_{g}$ and allowing the same transportation operator, with the corresponding list $V_{g}$ of vehicles in $\boldsymbol{T M}$ that can provide the trip from $d_{g}$ to $a_{g}$;

- all the subroutes $R_{\mathrm{g}}$ with $g \in\{1, \ldots, X\}$, defined as a five-tuple $R_{\mathrm{g}}=\left(d_{g}, a_{g}, W_{g}, u_{g}, V_{g}\right)$ where $\mathrm{d}_{\mathrm{g}}$ and $\mathrm{a}_{\mathrm{g}}$ are the departure and arrival nodes, $W_{g}=\left(t_{d g}, t_{a g}\right)$ is the couple of the departure and arrival times, and $\left(u_{g}, V_{g}\right) \in$ $\Lambda$ is the couple of the list of users that can use that route and the list of vehicles types in $\boldsymbol{T M}$ that can provide the trip;

- for each subroute $R_{\mathrm{g}}$ with $g \in\{1, \ldots, X\}$, the vehicle $V_{h, R_{g}}$ serving it;

- a matrix $\boldsymbol{W}=\left\{\mathrm{C}_{\mathrm{k}} w_{k \gamma}\right\} \in \Re \Re^{K \times c}$ (with $\Re$ the real numbers set) collecting weights $w_{k \gamma}$ associated with the $\gamma$-th 


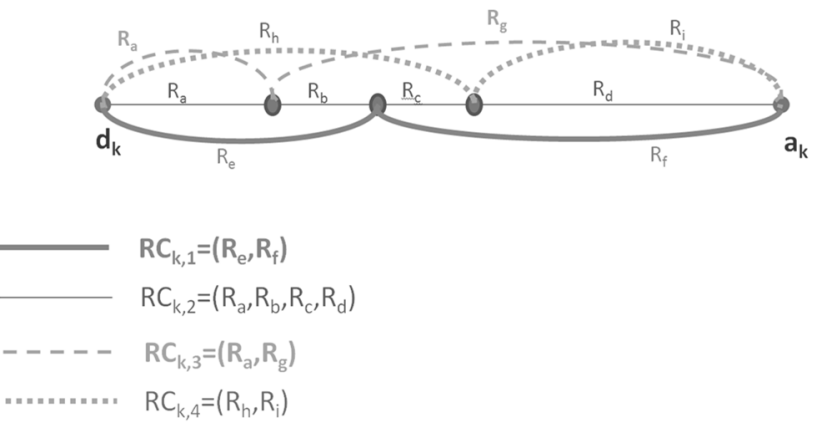

Fig. 3. Example of set of route combinations.

criterion for $k$-th user based on ordered list $C_{k}$ such that $\sum_{\gamma=1}^{c} w_{k \gamma}=1$.

The ATIS determines the recalled outputs from the given inputs by minimizing the fitness function defined as follows:

$$
\text { fitness } s_{k}=\sum_{\gamma=1}^{c} w_{k \gamma} \frac{\sum_{g} C_{\gamma, k, V_{h, R g}}}{\max _{g}\left(C_{\gamma, k, V_{h, R g}}\right)}
$$

for each request with $k=1, \ldots, K$ of the $k$-th solution $S_{k}$ - where $C_{\gamma, k, V_{h, R g}}$ is the $\gamma$-th performance index for subroute $R_{g}$ of the k-th solution with vehicle $V_{h, R_{g}} \sum_{\mathrm{i}=1}^{3} \mathrm{c}_{\mathrm{ki}}=1$.

\section{The Proposed Co-Modal Transport Criteria}

As mentioned earlier, in our specific problem, and with no loss of generality, we define $\mathrm{c}=3$ criteria that are the same for each k-th user (with different priorities): cost, time, and gas emissions. These are formulated as follows.

1) Transport cost for a subroute $R_{g}$. The cost of a subroute depends on the vehicle selected to serve it. In the case of public transport, the cost of trips is fixed and known in advance by the transport operator. In the case of carpooling and carsharing, the cost of a trip depends on the traveled distance. So, for the h-th vehicle which has to serve the subroute $R_{\mathrm{g}}$ (denoted by $V_{h, R_{g}}$ ) we have:

$$
C_{1, k, V_{h, R g}}=C_{1, V_{h, R g}}=C_{k m} \cdot f\left(V_{h, R_{g}}, D\left(d_{g}, a_{g}\right)\right)
$$

where it holds

$$
f\left(V_{h, R_{g}}, D\left(d_{g}, a_{g}\right)\right)= \begin{cases}D\left(d_{g}, a_{g}\right) & \text { for carsharing/ } \\ 1 & \text { poooling } \\ 1 & \text { otherwise }\end{cases}
$$

$C_{k m}$ is the unitary route cost (fixed for all public vehicles except for carpooling and carsharing where it equals that of a $1 \mathrm{~km}$ route) and $D\left(d_{g}, a_{g}\right)$ the distance between the starting point and ending point of the road in $\mathrm{Km}$, defined by their geographic coordinates (longitude and latitude).

2) Transport time for a subroute $R_{g}$. The transport time depends on the vehicle as follows. The travel time from

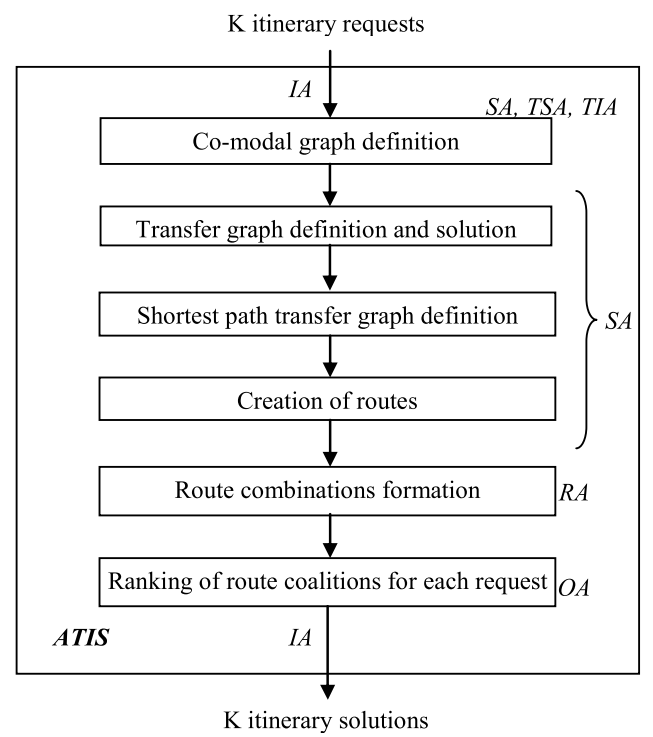

Fig. 4. The ATIS operations workflow.

the start point $d_{g}$ to the end point $a_{g}$ of the route $R_{\mathrm{g}}$ is denoted by $T\left(d_{g}, a_{g}\right)$ with:

$$
C_{2, k, V_{h, R_{g}}}=C_{2, V_{h, R_{g}}}=T\left(d_{g}, a_{g}\right)=\frac{D\left(d_{g}, a_{g}\right)}{V_{m}\left(V_{h, R_{g}}\right)}
$$

where $V_{m}\left(V_{h, R_{g}}\right)$ is the average speed of vehicle $V_{h, R_{g}}$ travelling along subroute $R_{\mathrm{g}}$.

3) Transport gas emissions for a subroute $R_{g}$. The calculation of the gas emission rate mainly depends on the traveled distance. We formulate this criterion as follows:

$$
C_{3, k, V_{h, R g}}=C_{3 V_{h, R g}}=D\left(d_{g}, a_{g}\right) \cdot F_{e}\left(V_{h, R_{g}}\right)
$$

with $F_{e}\left(V_{h, R_{g}}\right)$ the vehicle emission factor per $\mathrm{Km}$.

\section{The ATIS AGEnT-BASED ARCHITECTURE}

\section{A. The Proposed ATIS Workflow}

The workflow of the ATIS operations is shown in Fig. 4. The ATIS receives the requests by $K$ users. Hence, a domain search (explained in section IV and extended from [21]) is performed to identify the transportation means available for the requests and the co-modal graph representing the transportation network is built. Then, from the co-modal graph, a transfer graph is built, composed of a set of uni-modal networks called components, connected by arcs that model the change of transportation services. The transfer graph is solved by computing the intra-component paths, i.e., all the shortest paths (in terms of travel time) among particular points [43]. We remark that, in a transportation network, position points are represented by a number of nodes related by a set of vertices. In our work, a shortest path involves the minimum number of vertices between points. To find the shortest path between these points, the weight or length of a path is calculated as the sum of the weights of the edges in the path. So, a path is a shortest path if there is no path from the two points with lower weight. The shortest path is considered as a small size optimization problem, so we can use directly an exact method 
such as Dijkstra's algorithm to optimize locally criteria as the travel time, total travel cost, etc.

Here, for each obtained shortest path, a subroute is created: the combination of such routes is obtained; hence, the best solution for each request is calculated ranking such coalitions by a suitable performance index. More precisely, a genetic algorithm [28] is employed to select for each subroute in the paths the optimal configuration of user/transportation means association: this depends on the users' priority criteria, so that a multi-objective optimization is performed. After the genetic optimization, the best combination of subroutes provides the ATIS response to the users. Each flowchart step can involve one or several agents, presented in the sequel.

\section{B. The ATIS Multi-Agent Architecture}

The paradigm used to implement the ATIS is that of MultiAgent Systems (MAS) [34]. An agent is a system perceiving the environment by sensors and impacting it by actuators. A MAS is a system made by several agents interacting with each other. In MASs, two main categories of interactions among agents exist: cooperation and negotiation. Negotiation occurs in case of conflictual goals (antagonist agents). Cooperation is a form of interaction between autonomous and rational entities, called agents, to jointly solve tasks or maximize utility. This notion is one of the key concepts differentiating MASs from other related disciplines such as distributed computing, object-oriented systems, and expert systems. Further, each agent is composed of states, different types of knowledge (environmental, social and personal), messages, behavior rules (i.e., a list of tasks) and a perception function. Thanks to the Behavior rule, the agent can change state according to current states, knowledge and received messages in order to reach the collective goal. A set of rules and behaviors can define a role. An agent can have different roles, but it can run just one at time. From a role to another, the agent changes its capabilities and behaviors. Each agent knows only its information. To exchange the information and achieve a desired global goal, agents can send and receive messages labeled MESSAGE between each other.

The ATIS architecture is described by Fig. 5, including: Interface Agents (IA) receiving users' requests; a Super Agent (SA) building and solving the co-modal graph and creating the Route Agents (RA); Transport Service Agents (TSA) searching for transportation means suitable for the requests; Transport Information Agents (TIA) communicating with the transportation operators database; RAs building subroute coalitions; an Optimizer Agent (OA) calculating the best solution for each user request. Accordingly, the workflow in Fig. 4 reports the agents in the different operations.

We now detail the MAS implementation of the ATIS. A TSA $T S_{i}$ with $i \in\{1, \ldots, n\} \operatorname{TSA}_{\mathrm{i}}, 1 \leq \mathrm{i} \leq \mathrm{K}$ is associated to each transport service and a TIA $T I_{i j}$ with $i \in\{1, \ldots, n\}$ and $j \in\left\{o_{1}, \ldots, o_{n}\right\}$ is associated to each transport operator. Generally, a TSA is responsible for a set of TIAs. Each TIA is able to respond to an itinerary request $i_{k}$ with $k \in\{1, \ldots, K\}$ by proposing some routes of the transportation network that can satisfy the user requests. For each request, an IA $I A_{k}$ with $k \in\{1, \ldots, K\}$ interacts with the $k$-th user to take into

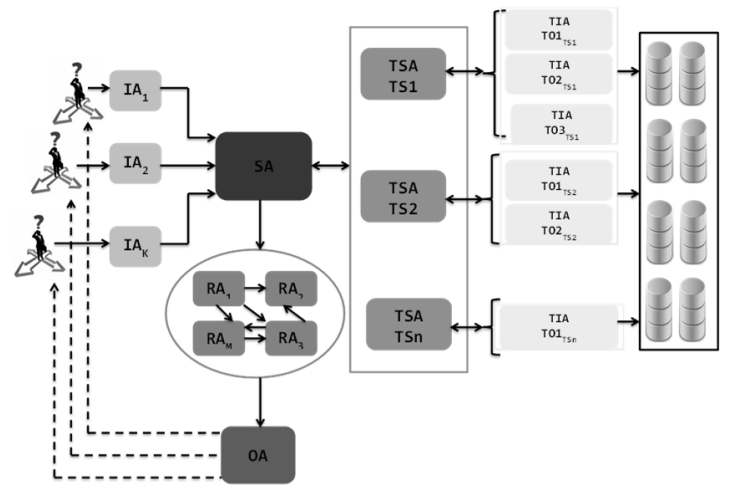

Fig. 5. The ATIS multi-agent architecture.

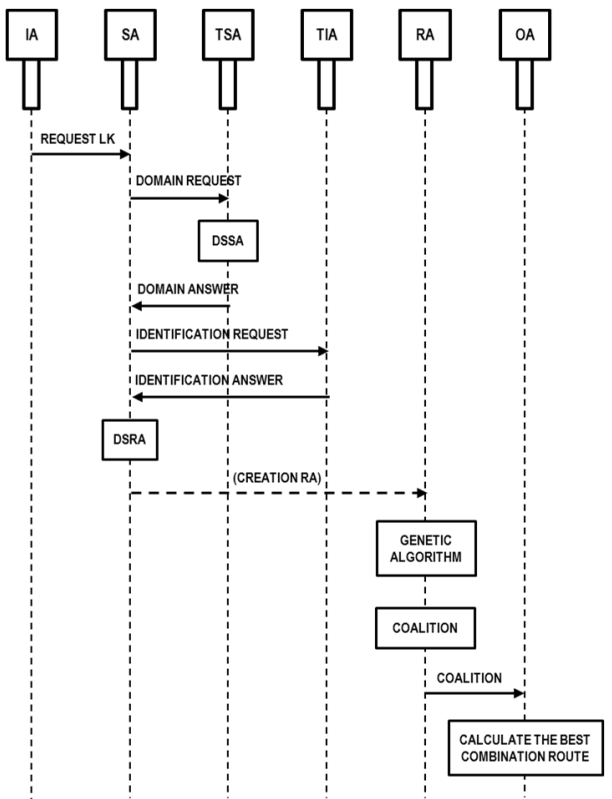

Fig. 6. Sequence diagram and message exchange of the multi-agent ATIS.

account its preferences and constraints and sends a message to the SA. This message corresponds to request $i_{k}$ of the sequence diagram in Fig. 6, showing the exchange of messages and main operations of the ATIS agents. As shown in Figs. 5 and 6, the SA plays several roles. First, it communicates with the agents describing the transportation services and operators; the goals of these communications are finding a suitable transportation means for the request and constructing the co-modal graph. To achieve this goal, the SA sends the message DOMAIN_REQUEST to the TSAs that each run the Domain Search Selection Algorithm (DSSA, detailed in section IV) and answers with a message DOMAIN_ANSWER (Fig. 6): these communications provide the SA with the information about the transportation means available for the set of requests. Second, the SA communicates with the TIAs by the messages IDENTIFICATION_REQUEST and IDENTIFICATION_ANSWER (Fig. 6) in order to collect the information about the sub-networks of transportation means available for the requests. Then, with this information the SA creates and solves the co-modal graph (block DSRA or Distributed 
Shortest Route Algorithm in Fig. 6), as described in the next section. Third, the SA generates a set of RAs (\{creation $R A\}$ in Fig. 6). Each RA represents a generated chromosome scheme for an identified subroute $R_{\mathrm{g}}$ in order to assign concerned users to possible vehicles (see the subsequent section). As soon as each RA assigns persons to vehicles, updating the number of passengers in private pooling vehicles and the number of available vehicles of public sharing services, it computes all values criteria of each vehicle for each assignment. A multiagent coalition [14], [41] is then created regrouping all RAs corresponding to a possible route combination for a given itinerary. Therefore, we have as many coalitions as combinations knowing that an RA can belong to many different coalitions according to combinations overlapping. Coalitions appear and disappear dynamically according to requests. Then, the generated data is transferred by the coalition message of Fig. 6 to the OA who decides the best combinations thanks to its interaction with the autonomous RAs. The OA computes the best route combination for each itinerary demand according to the users' criteria and constraints by ranking solutions with a suitable performance index and sends it to the corresponding IA.

\section{Description of the ATIS's Agents}

As specified earlier, the IA is the agent that communicates with users to read their requests, i.e., the five-tuples described in section 2. Unlike our previous work [21], through the ATIS interface it is possible to choose also the preferences of the criteria: the weight of each criterion for the optimization phase is assigned according to this choice. This agent has a simple behavior: it receives the requests and it sends them to the SA.

The SA is the ATIS core and the most complex agent, having three roles (Fig. 7). The first role is to define the domain search by communicating with the TSAs that in turn each communicate with their TIAs. The SA sends a message to the TSAs, with the departure and arrival points of the requests that it received by the IAs.

Each TSA executes the Domain Search Selection Algorithm (DSSA, detailed in section IV) and it answers to the SA with a list of possible transportation means that can be involved in the itineraries.

To this aim, each TSA sends a message to the TIAs that in turn answer with some graphs describing a piece of the transportation network concerning some means of transport. After receiving the DSSA results, in its second role the SA builds from the received graphs the co-modal graph to represent the transportation network. Hence, in its third role is to determine the subroutes to be composed into the final itinerary by generating multiple RA.

The TSA is the agent that represents a type of transportation service: it manages a pool of TIAs to provide to the SA the list of transportation means to respond to the requests. This agent receives the request from the SA with the departure and arrival points and by running the DSSA, it identifies the set of transportation means to be involved to provide the possible available subroutes and satisfy the user request.

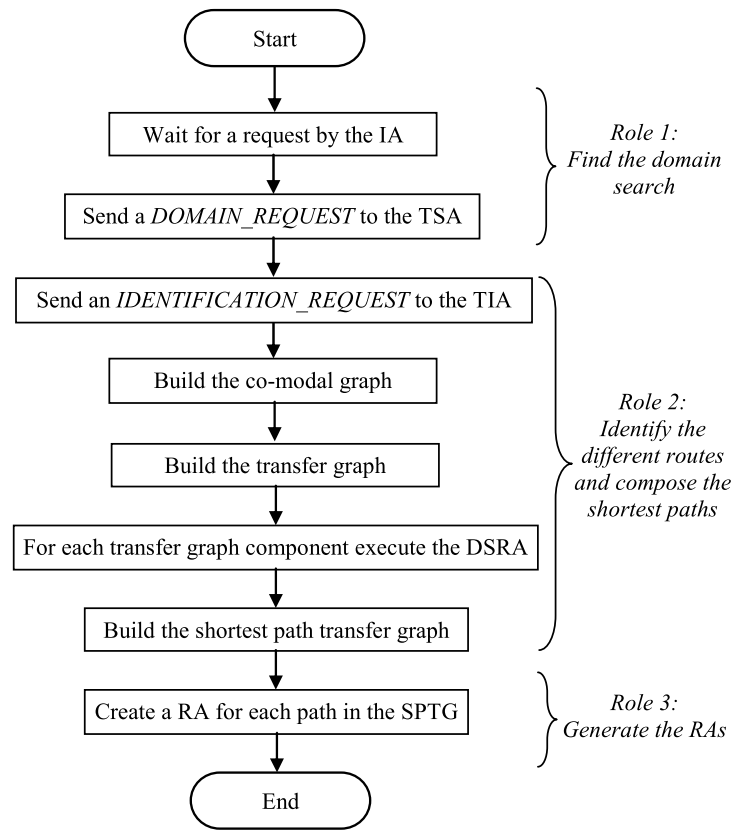

Fig. 7. The Super Agent's flowchart.

Each TIA represents one transport operator: so each agent is responsible for its network operators. It receives the requests by the SA; these requests, for each concerned TIA, include the names of involved means of transport and departure and arrival times. The TIA accesses its database, recovers the points of the network and admissible departure times for each point, and provides the SA with a graph. For each edge of this graph there is a list of departure and arrival times, including all times in the given time window. Each TIA sends its graph to the SA, which composes these sub-graphs into the complete transportation network graph. Since the optimization of the graph concerns a criterion optimization, i.e., searching for the best path in terms of time, the edges weights are given by the travelling time between two points.

Using the results of the DSSA instances by the TSAs it has communicated with, the SA represents the transportation network by a directed graph called co-modal graph (defined in the subsequent section) that describes the given transportation network. Starting from the co-modal graph, a transfer graph (defined in the subsequent section) is obtained to represent the distributed nature of the transportation network, separating this into different uni-modal networks where one type of transportation service is available (e.g., public transport, private vehicle pooling, public vehicle sharing). Hence, the DSRA is executed by the SA for each determined uni-service network and the results are employed to build a Shortest Path Transfer Graph (SPTG, defined in the subsequent section).

Using the SPTG, the SA in its third role (Fig. 7) generates the multiple RAs for further combinations or coalitions in order to determine the final itinerary solution. The RA represents a subroute, identified by the departure and arrival points. More precisely, after the application of the DSRA, the SA represents each subroute in the paths by a RA that is a matrix where rows correspond to users and columns are the different vehicles serving the subroute. 


\begin{tabular}{cccccc}
\hline \hline \multicolumn{5}{c}{ Bike 12Subway 2Train 1Bus 9Car 7 } \\
\hline \hline $\mathrm{i}_{2}$ & $\mathrm{x}$ & $\mathrm{x}$ & $\mathrm{x}$ & 1 & ${ }^{*}$ \\
\hline $\mathrm{i}_{3}$ & $*$ & 1 & ${ }^{*}$ & $\mathrm{x}$ & ${ }^{*}$ \\
\hline $\mathrm{i}_{6}$ & $*$ & $\mathrm{x}$ & $\mathrm{x}$ & ${ }^{*}$ & 1 \\
\hline $\mathrm{i}_{15}$ & $\mathrm{x}$ & $*$ & 1 & $\mathrm{x}$ & ${ }^{*}$ \\
\hline \hline
\end{tabular}

Fig. 8. Example of a chromosome solution.

\section{The User/Vehicle Assignment by Genetic Optimization}

The final user/vehicle assignment is made by a Genetic Algorithm (GA). GAs are parallel stochastic search methods to solve problems, imitating Darwinian evolution laws [28], based on a simple iterative scheme. The reason for this choice is the diversity and the efficiency of population-based techniques: a GA initializes and progressively let evolve a population of solutions of the problem appropriately encoded in a pre-selected alphabet. The fitness of each individual (solution) in the population is then computed. The fitness value rules the iterative search schema, which is performed in two subsequent phases: selection and recombination. After these, a new population (offspring) is ready for fitness evaluation. By iteration of this search schema, the GA converges toward better solutions in a wide class of hard numerical optimization problems.

The key feature of a GA is coupling the global optimization done by the genetic operators and the local search that enhances the explored solutions. The population of chromosomes evolves throughout a series of controlled crossovers and mutations operators. In particular, the GA uses the classical roulette wheel selection and standard crossover and mutation operators, applied with probabilities $p_{\text {cross }}=0.5$ and $p_{m u t}=$ 0.002 respectively. These generate feasible offsprings thanks to a correction process that allows avoiding the assignment of multiple transportation means for the same subroute and the same user. For more details on the GA and such a correction process, the reader is referenced to [20].

Each chromosome of the population can represent one or multiple solutions depending on the used encoding. A direct encoding means that the solution is directly represented by the chromosome and that no decoding procedure is needed. An indirect encoding means that a decoding procedure is needed to retrieve the solution from the chromosome. We use a direct coding chromosome, which gives us a complete solution to be directly exploited by the manager of the co-modal transport network. Therefore, the decoding process is not necessary in our approach, which reduces the computation time.

Hence, in our case, the ATIS GA result is a feasible and effective user/vehicle assignment that minimizes the objective function (see the example in Fig. 8). The chromosome is a matrix $\mathbf{C H}$ where rows correspond to users and columns to identified vehicles $\mathrm{V}_{\mathrm{h}}^{\mathrm{Rg}}$ available through the time window $\mathrm{W}_{\mathrm{g}}$ to serve the same subroute $R_{\mathrm{g}} \mathrm{R}_{\mathrm{g}}\left(\mathrm{d}_{\mathrm{g}}, \mathrm{ag}_{\mathrm{g}}, \mathrm{W}_{\mathrm{g}}\right)$. Hence, each element of the matrix is an assignment of the user $k$ to the vehicle $h$ as follows:

$$
C H(k, h)= \begin{cases}1 & \text { if user } \mathrm{k} \text { is assigned to vehicle } \mathrm{h} \\ * & \text { if user } \mathrm{k} \text { can be assigned to vehicle } \mathrm{h} \\ \mathrm{x} & \text { if user } \mathrm{k} \text { cannot be assigned to vehicle } \mathrm{h} .\end{cases}
$$

Given the $\boldsymbol{C H}$ matrix of the RA, the GA checks for each row whether there is one and only one assignment user-means of transport (i.e., one 1 element): if this condition is not verified, it performs selection and recombination operations on matrix $\boldsymbol{C H}$ to retrieve a correct configuration. After the departure and arrival times' choice, the best solution is calculated for each request. The fitness for each solution for the $k$-th user is defined by equation (1) in section II.

Afterwards, coalitions take place (see the subsequent section). In general, there are a few RAs, with the departure flag active, that start the coalition. They send a coalition request to all the RAs linked with them; the agent that accepts the request sends a request to all its neighboring agents. The construction of coalitions finishes after a predefined time. While the ATIS continues to identify the best route combinations thanks to the GA and the RAs coalition, the SA keeps in mind all these routes. We plan in future research to allow the RAs coalition consult the SA and its knowledge, since it could optimize the number of messages and negotiation between the different RAs. In this paper, we do not detail the RAs coalition and the negotiation protocol but we focus on the distributed co-modal approach used in order to compute the shortest paths in the co-modal graph.

After the coalitions' construction, the agents with arrival flag active send the coalitions to the OA. The OA is the last agent of the ATIS architecture and it is devoted to the calculation of the best solution for each request. The calculation of the best solution is referred to a single user: the value of a solution is performed only on the row of the chromosomes related to that user. For each route and for each user, the OA evaluates fitness $\mathrm{k}_{\mathrm{k}}$ choosing the best route combination and sending the answer to the user.

\section{The Distributed Co-Modal OPTIMIZATION APPROACH}

Determining the solution to requests involves three steps.

The first step is the identification of the research area or domain search. It is devoted to determining the set of available transport operators, whatever their service, and providing all data and possible routes that can build global solutions. The second step is to look for different subroutes which may constitute the final solutions.

We start with a single-criterion optimization in terms of time, by running a distributed Dijkstra algorithm applied to a transfer graph. The transfer graph is a specific graph composed of a set of components connected with a set of virtual arcs. Each component represents a transport service. In the transfer graph, we distinguish between two path types: intercomponents and intra-components [21]. An inter-component path is any path connecting two vertices, where at least two edges belong to two distinct components. On the contrary, an intra-component path connects two vertices whose edges belong only to a single component. Figure 9 gives an example of a 3-component transfer graph: carpooling, public transport and free use vehicle. Thus we get the shortest paths in each component, enabling us to generate a set of RAs.

The third step applies a genetic approach to each RA to determine the best routes for each request in terms of the 


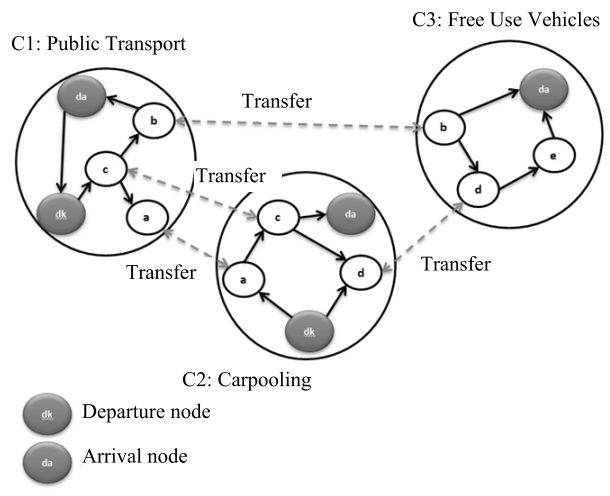

Fig. 9. Example of a transfer graph.

three criteria. Following this approach, communication between RAs through the coalition to compose the co-modal routes optimized. EA agent calculates finally the best combination of routes for each request and forwards it to the user.

\section{A. The Domain Search}

The domain search is performed by a TSA to select the means of transport: to search for these, it is necessary to know the departure and arrival points for each user's submission. To achieve this goal, we use a directed graph called Adjacency Graph $\boldsymbol{G}_{\mathrm{A}}=\left(\boldsymbol{N}_{\mathrm{A}}, \boldsymbol{E}_{\mathrm{A}}\right) \mathrm{G}(\mathrm{N}, \mathrm{E})$, where the set of nodes $\boldsymbol{N}_{\mathrm{A}}$ represents the set of the transportation means of the TSA operators and the set of edges $\boldsymbol{E}_{\mathbf{A}}$ connects all couples of nodes, representing the fact that two vehicles share (do not share) a vehicle stop, where it is possible (not possible) to change vehicle by an arc weight that is equal to $1(0)$ when that link exists. The domain search is executed by the DSSA on the adjacency graph: the inputs of the algorithm are the departure and arrival points and the adjacency graph. The outputs are a certain number of paths: this number can be changed. The goal is to search for the paths with minimal weight linking departure and arrival points. Therefore, the algorithm includes an identification procedure: transportation means that include departure and arrival points are searched for. The nodes of the graph referred to vehicles that include departure points are classified as departure nodes, while the nodes referred to vehicle that include arrival points are classified as arrival nodes. After this identification part, the algorithm is executed. The calculation of paths is performed by the wellknown Dijkstra Algorithm: after a computation, the weight of arcs included in the minimal paths increases of 1 . The Dijkstra Algorithm works iteratively but the new computation is calculated on the new adjacency graph (with the increased weights). The algorithm stops when the number of paths is reached or after a certain number of iterations without any new solution. In [22], the algorithm for the domain search is applied to the TIA domain instead of the transportation means domain: the output of the algorithm is a list composed by the names of the TIAs. With this list we have to communicate with all transportation means of the involved TIA. However, if the algorithm is applied to the transportation means domain, as we do in this work, the resulting list allows communicating directly with the involved means of transport.
For the sake of brevity, we avoid reporting the complete DSSA algorithm, which may be found in full detail in [22].

\section{B. The Co-Modal Graph and the Transfer Graph}

The SA represents the transportation network by a directed graph $\boldsymbol{G}_{\mathbf{C}}=(\boldsymbol{N}, \boldsymbol{E}, \boldsymbol{T S})$ that describes the given transportation network, where $\boldsymbol{N}=\left\{n_{1}, \ldots, n_{\mathrm{j}}, \ldots, n_{\mathrm{J}}\right\}$ is a set of vertices that are $J$ in number, $\boldsymbol{E}=\left\{e_{1}, \ldots, e_{l}, \ldots, e_{L}\right\}$ is a set of edges that are $L$ in number, and $\boldsymbol{T S}=\left\{T S_{1}, \ldots, T S_{i}, \ldots, T S_{n}\right\}$ is the set of transportation services (e.g. public transport, vehicle sharing and private vehicles), with $\mathrm{n}$ their total number [21]. An edge $e_{l} \in E$ is defined by $\left(\left(n_{p}, n_{q}\right), T S_{i}\right)$ where $n_{p}, n_{q} \in N$ and $T S_{i} \in \boldsymbol{T S}$. Hence, edge $e_{l}$ expresses the fact that it is possible to move from vertex $n_{\mathrm{p}}$ to $n_{\mathrm{q}}$ by using the transportation service $T S_{i}$. A weight $D e_{1}$ is associated to each edge $e_{1} \in \boldsymbol{E}$, indicating the cost of including the edge in the solution. The transportation network directed graph is called a co-modal graph if and only if it includes at least two transport services, i.e., $n>1$ and $\exists T S_{i}, T S_{i^{\prime}} \in \boldsymbol{T S}$ where $\left(\left(n_{p}, n_{q}\right), T S_{i}\right),\left(\left(n_{p}^{\prime}\right.\right.$, $\left.\left.n_{q}^{\prime}\right), T S_{i}{ }^{\prime}\right) \in \boldsymbol{E}$ and $T S_{i} \neq T S_{i^{\prime}}$. On the contrary, if there is just one transportation service, then the graph is said to be uniservice. Given a co-modal graph $\boldsymbol{G}_{\mathbf{C}}=(\boldsymbol{N}, \boldsymbol{E}, \boldsymbol{T S})$, a route combination $R C_{n_{\mathrm{h}} n_{1}}=\left(n_{\mathrm{h}} \rightarrow n_{1}\right)=\left(e_{\mathrm{h}}, e_{2}, \ldots, e_{1}\right)$ is a sequence of edges or subroutes between a pair of vertices $n_{\mathrm{h}}$ and $n_{1}$.

A path $R C_{n_{\mathrm{h}} n_{1}}$ is said co-modal if it includes two edges $e_{\mathrm{p}}, e_{\mathrm{q}} \in R C_{n_{\mathrm{h}} n_{1}}$ with $\mathrm{e}_{\mathrm{p}}=\left(\left(n_{\mathrm{p}}, n_{\mathrm{p}^{\prime}}\right), T S_{i}\right), e_{\mathrm{q}}=\left(\left(n_{\mathrm{q}}, n_{\mathrm{q}^{\prime}}\right), T S_{j}\right)$, $T S_{i} \neq T S_{j}$. Instead, if only one service is involved, the path is said to be uni-service. Figure 10. shows a co-modal graph and its transportation services (train, bus, bike sharing, car sharing, private carpooling). In [4], an approach based on the transfer graph is proposed for a time-dependent multimodal transport problem. Here, we extend the work in [4], proposing a transfer graph in which the weight of the arcs is not timedependent, but the graph itself can change according to time. In particular, we start from a co-modal graph that includes $n$ transportation services: hence, we can decompose the graph into one transfer graph including $n$ uni-service networks connected by arcs modeling the change of transportation services.

Accordingly, the transfer graph is defined as $\boldsymbol{G}_{\mathbf{T}}=(\boldsymbol{C C}$, $\boldsymbol{T R})$ where $\boldsymbol{C C}=\left\{\boldsymbol{C} \boldsymbol{C}_{\mathbf{1}}, \ldots, C C_{i}, \ldots, \boldsymbol{C C _ { \mathbf { r } }}\right\}$ is the set of uniservice networks called components and $\boldsymbol{T R}$ is the set of virtual edges which interconnect them. In particular, each component $\boldsymbol{C} \boldsymbol{C}_{\mathbf{i}}=\left(\boldsymbol{N}_{\mathbf{i}}, \boldsymbol{E}_{\mathbf{i}}, T S_{i}, \boldsymbol{P} \boldsymbol{T} \boldsymbol{C}_{\mathbf{i}}\right)$ is such that $\forall i, j \in\{1, \ldots, r\}$ it holds $T S_{i} \neq T S_{j}$. Besides, it holds $\mathbf{N}=\left\{N_{i} \mid i \in\{1, \ldots, r\}\right\}$, $\mathbf{E}=\left\{E_{i} \mid i \in\{1, \ldots, r\}\right\}, \mathbf{T S}=\left\{T S_{i} \mid i \in\{1, \ldots, r\}\right\}$, and $\mathbf{T R}=\left\{\left(n_{i}, n_{j}\right) \mid n_{\mathrm{i}} \in \boldsymbol{C}_{\mathbf{i}}, n_{\mathrm{j}} \in \boldsymbol{C} \boldsymbol{C}_{\mathbf{j}}, i \neq j, n_{\mathrm{i}}=n_{\mathrm{j}}\right\}$ where $\left(n_{\mathrm{i}}, n_{\mathrm{j}}\right)$ represents a change of transport service from $T S_{i}$ to $T S_{j}$ at the co-modal transfer point $n_{\mathrm{i}}$ (or equally $n_{\mathrm{j}}$ ). In particular, $n_{\mathrm{i}} \in \boldsymbol{P} \boldsymbol{T} \boldsymbol{C}_{\mathbf{i}}, n_{j} \in \boldsymbol{P} \boldsymbol{T C} \boldsymbol{C}_{\mathbf{j}}$, with $i, j \in\{1, \ldots, r\}$ are called co-modal transfer points and are symbolized by the same location. Hence, for each component $\boldsymbol{P} \boldsymbol{T} \boldsymbol{C}_{\mathbf{i}}=\left\{n_{\mathrm{i}} \in \boldsymbol{C} \boldsymbol{C}_{\mathbf{i}} \mid\right.$ $n_{j} \in \boldsymbol{C} \boldsymbol{C}_{\mathbf{j}}$ with $\left.\mathrm{n}_{\mathrm{i}}=\mathrm{n}_{\mathrm{j}}, i, j \in\{1, \ldots, r\}\right\}$ is the set of co-modal transfer points.

We remark that the weight of an arc in the transfer graph corresponds to the cost of the path between two nodes, just as 


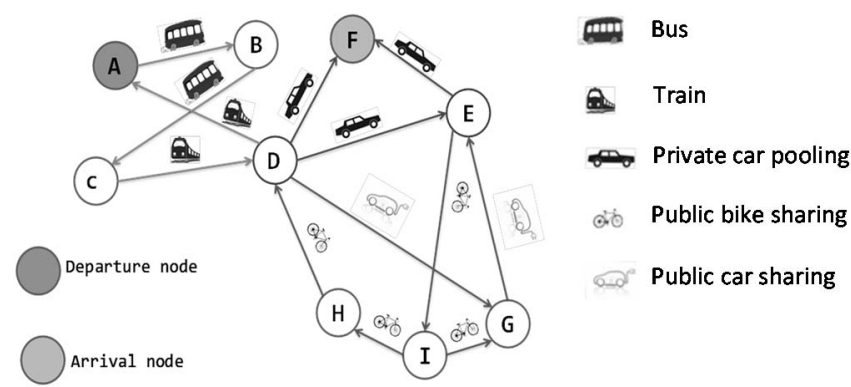

Fig. 10. Example of co-modal graph with five transportation services.

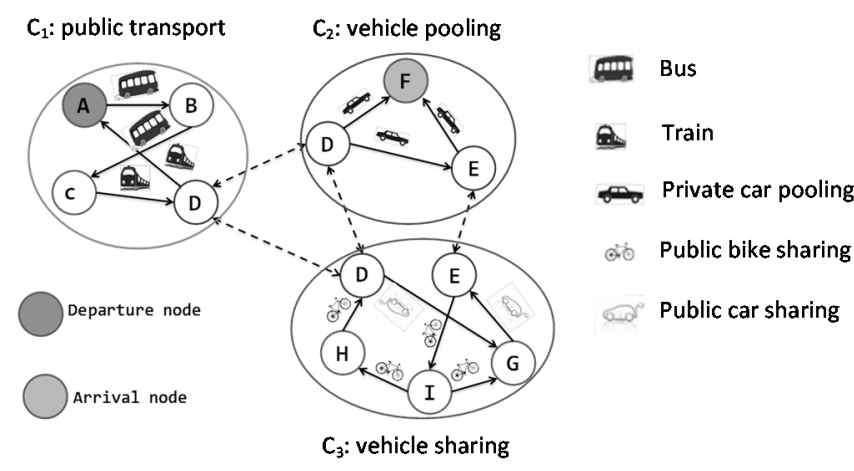

Fig. 11. Example of transfer graph with five components.

in the co-modal graph, since the transfer graph is a deduction of the co-modal graph (same nodes and same edges linking these nodes). The cost of an edge integrates $\mathrm{c}=3$ attributes, corresponding to the $c$ criteria: cost, time, and emissions. These will be aggregated through the optimization phase.

Summing up, the transfer graph represents the distributed nature of transportation. It allows separating all transportation modes in the network, obtaining different so-called uni-modal or uni-service networks: so, each component or uni-modal network is independent and can be easily changed or updated without requiring any further recalculation [43]. Figure 11 shows an example of transfer graph obtained from the co-modal graph in Fig. 10, with five transportation services, three components (public transport, vehicle pooling, vehicle sharing) and two co-modal transfer points $(D, E)$.

As recalled earlier, an intra-component path of a component $\boldsymbol{C} \boldsymbol{C}_{\mathbf{i}}=\left(\boldsymbol{N}_{\mathbf{i}}, \boldsymbol{E}_{\mathbf{i}}, T S_{i}, \boldsymbol{P T C} \boldsymbol{C}_{\mathbf{i}}\right)$ can be one of the following types [21]:

- $R C *_{i, d_{k}, a_{k}}$ is the shortest path which starts at source vertex $d_{k}$ and ends at target vertex $a_{k}$ within $\boldsymbol{C C}_{\mathbf{i}}$;

- $R C *_{i, d_{k}, P T C_{i}}$ is the shortest path starting at source vertex $d_{k}$ and ends at a co-modal transfer point $\boldsymbol{P} T \boldsymbol{C}_{\mathbf{i}}$ within $C C_{\mathrm{i}}$;

- $R C *_{i, P T C_{i}, P T C_{j}}$ is the shortest path which starts at any co-modal transfer point $\boldsymbol{P T \boldsymbol { C } _ { \mathbf { j } }}$ and ends at a co-modal transfer point $\boldsymbol{P} \boldsymbol{T} \boldsymbol{C}_{\mathbf{i}}$ within $\boldsymbol{C} \boldsymbol{C}_{\mathbf{i}}$;

- $R C *_{i, P T C_{j}, a_{k}}$ is the shortest path starting at any co-modal transfer point $\boldsymbol{P} \boldsymbol{T} \boldsymbol{C}_{\mathbf{j}}$ and ending at target a $\mathrm{a}_{\mathrm{k}}$ within $\boldsymbol{C} \boldsymbol{C}_{\mathbf{i}}$.

The transfer graph $\boldsymbol{G}_{\mathbf{T}}=(\boldsymbol{C C}, \boldsymbol{T R})$ can thus be solved by computing all the different intra-component paths of the four types listed above. The computation of these paths is made using the DSRA [43]. In this paper, we use a variant of this algorithm: we execute the DSRA in parallel, for each uni-service network: we make a clone of the SA for each component of the transfer graph; each clone executes an instance of the DSRA referred to one single component.

Then, the clones send the results to SA that builds the Shortest Path Transfer Graph (SPTG). Given a transfer graph $\boldsymbol{G}_{\mathbf{T}}=$ $(\boldsymbol{C C}, \boldsymbol{T R})$, we define SPTG a graph $\boldsymbol{G}_{\mathrm{SPT}}=\left(\boldsymbol{N}_{\mathrm{SPT}}, \boldsymbol{E}_{\mathrm{SPT}}\right)$, where $E_{S P T}=\bigcup R C *_{i, d_{k}, a_{k}} \bigcup R C *_{i, d_{k}, P T C_{i}} R C *_{i, d_{k}, a_{k}} \bigcup$ $R C *_{i, d_{k}, P T C_{i}} \bigcup R C *_{i, P T C_{i}, P T C_{j}} \bigcup R C *_{i, P T C_{j}, a_{k}} \forall \boldsymbol{C} \boldsymbol{C}_{\mathbf{i}} \in \boldsymbol{C C}$, $\boldsymbol{N}_{\mathbf{S P T}}=\left\{n \mid \exists e \in \boldsymbol{N}^{+}(\boldsymbol{n}), e \in \boldsymbol{E}_{\mathbf{S P T}} \vee \varepsilon \in \boldsymbol{N}^{-}(\boldsymbol{n}), \varepsilon \in \boldsymbol{E}_{\mathbf{S P T}}\right\}$.

For the sake of brevity, we avoid reporting the complete DSRA algorithm, which may be found in full detail in [21].

\section{The Subroutes Coalition Into Routes}

After the GA is executed and terminated by a RA, coalitions are built. A coalition is an ordered succession of RAs.

The solutions to the users' requests can be obtained by the communication of RAs; each RA represents a piece of the whole path that is crossable with at least one transportation mean. Each RA communicates with its neighbor agents to achieve the coalitions. With these alliances, we want to obtain every possible combination of subroutes; among these combinations we search for the best solution.

Every RA has two lists: the neighbor receivers list, with the names of the RAs that have an arrival point equal to the its departure point; the neighbor senders list, with the names of RAs that have a departure point equal to the its arrival point.

A RA can have two particular roles: if its departure flag is active, the RA is called initiator agent and it starts the coalition; if its arrival flag is active, the RA is called terminator agent and when the building of the coalitions is finished, it sends the results to OA.

Independently by their classification, every RA performs the following steps (because it is possible that these agents are not initiator or terminator for other requests):

While (neighbor receivers not empty and time not expired):

1) Receive a message;

2) Verify if sender wants to be deleted: if it is verified, delete sender from the neighbor receivers;

3) Verify if message is valid: if it is valid, send a message to each other neighbor sender agents; if neighbor receivers list is empty, communicate to the agents of neighbor senders list that RA wants to be deleted.

Only the initiator agents can start the coalition; these agents insert in the message of coalition their names and their users which have a departure point of their request equal to the departure point of the subroute. A RA participates in the alliance if and only if it receives a message; this message can be sent by an initiator agent or by an agent that has received the starting message by an initiator agent. When a RA receives a message, before it is accepted, it controls two conditions that have both to be valid: its arrival point is not already in the coalition; it has at least a valid user for this coalition.

When a RA sends a message, if its neighbor receivers list is empty, the RA sets a parameter of the message. When the agents receive this message with the parameter set, they delete 
TABLE I

COMPARISON OF THE TSP With THE Co-Modal TRANSPORT Problem

\begin{tabular}{lll}
\hline \hline Problem & $\begin{array}{l}\text { Traveling } \\
\text { Salesman Problem } \\
\text { (TSP) }\end{array}$ & $\begin{array}{l}\text { Co-modal transport } \\
\text { problem (for a single } \\
\text { transport request) }\end{array}$ \\
\hline Parameter & $\begin{array}{l}\text { Traveler's passing } \\
\text { cities. }\end{array}$ & $\begin{array}{l}\text { Correspondence nodes. } \\
\text { Constraint }\end{array}$ \\
$\begin{array}{l}\text { Each city must be } \\
\text { visited exactly once } \\
\text { by only one traveler. }\end{array}$ & $\begin{array}{l}\text { Eust visit only once each } \\
\text { correspondence node, } \\
\text { respecting delays. }\end{array}$ \\
& $\begin{array}{l}\text { Find the optimized } \\
\text { tour: the shortest } \\
\text { path connecting all } \\
\text { cities. }\end{array}$ & $\begin{array}{l}\text { User satisfaction: providing } \\
\text { according to the preferences } \\
\text { and requirements of the } \\
\text { user. }\end{array}$ \\
\hline \hline
\end{tabular}

the sender of the message from their neighbor receivers list. An agent finishes the building process when the neighbor receivers list is empty or the execution time is expired; this time is editable. When a terminator agent finishes this behavior, it sends its list of the solutions to the OA. When all the terminators finish the construction of the solutions, the OA starts the computation of the best solution for each request.

\section{The ATIS Scalability}

The proposed ATIS approach is scalable thanks to the dynamicity of the agents' creations. Indeed, the SA creates and destroys dynamically the RAs according to the users' requests. After the creation, the SA assigns to each RA a subroute to optimize it locally. Each RA uses a GA to choose the best means of transport that will be used to serve locally this route for each user. Hence, each RA handles an initial population with a reasonable number of chromosomes to accelerate the convergence. In addition, all the RAs work in parallel to find locally their optimal routes respectively and collaborate with the other RAs forming coalitions in order to build the optimal global route for each request.

\section{E. The ATIS Complexity and Convergence}

According to the described formulation, the ATIS receives a set of simultaneous requests. To treat them, the ATIS receives information from different transport operators of different transport services. All these responses form a co-modal graph representing the map of the routes of different transport services. A co-modal graph is dense and contains a lot of nodes, whether connecting nodes for transit, appointments nodes for carpooling or nodes representing vehicle stations selfservice. In the literature, this problem is similar to the wellknown Traveling Salesman Problem (TSP). In this context, we propose a comparative study of the optimization problem of a co-modal transport system and the TSP (Table I). In short, given a co-modal graph $\mathrm{G}$ with $n$ correspondence nodes, a number of $(n-1) ! / 2$ itineraries is possible. If we consider that the time itinerary evaluation has a complexity $\mathrm{O}(n)$, we obtain a total time complexity of $\mathrm{O}(n !)$ : for 6 nodes, we have 72 . possibilities, for 10 nodes, 3628800 possibilities.
According to this analogy, the TSP is similar to one instance of our co-modal transport problem (a single user request). However, this comparison takes into account a single user request at time $t$. Instead, in our co-modal transport ATIS, we can receive at time $t$ a set of simultaneous requests. This increases considerably the complexity of the problem in comparison with the TSP problem. We conclude that the ATIS transport co-modal problem is a highly combinatorial optimization problem.

To counteract for this complexity, which increases proportionally with the transportation network size, the architecture of ATIS is based on a collaborative optimization, using the alliance between the meta-heuristics and the multi-agent systems. This alliance is an original approach, integrating GAs with autonomous and intelligent agents. These entities work together in a parallel and distributed manner through interaction protocols, by forming intelligent coalitions in order to solve co-modal itineraries problems.

The main difficulty in the use of multi-agent systems is to control the behavior and the evolution of the overall system consisting of autonomous agents: for a given problem, it is not assured that all agents converge to a single common solution. Hence, the proposed ATIS has a distributed architecture consisting of several route agents, which cooperate and interact through an exchange of messages, according to a decentralized approach to build a local route within each agent, so as to converge at best towards a global solution that meets the requirements adopted collectively (global itineraries). In fact, in a society of agents, initially each agent behaves according to an initial strategy, but with passing time, global phenomena emerge that most agents tend to follow, thus converging towards a behavioral strategy called collective synchronization (see the discussions in [14] and [41]).

\section{The Case Study}

The ATIS is developed on the JADE platform in the Java agent framework [5]: JADE is a middleware allowing a flexible implementation of multi-agent systems and offering efficient agent communication. Moreover, the ATIS is implemented based on the real road network using Google Maps and its geolocation application programming interface. The user path, composed by several subroutes, is printed on a static Google map [15]. Using Google Maps, travel distances and times are obtained for origins and destinations.

The ATIS is tested for the Lille area (France, Fig. 12) by an extra-urban and urban scenario for $n=3$ transportation services: $\mathrm{TS} 1=$ public transportation; TS $2=$ vehicle sharing; TS3 $=$ private vehicles (car pooling). Operators are: TO1TS1=Transpole [38], with subway lines 1, 2 and urban buses 43, 44; TO2TS1=S.N.C.F. [36] with trains Ter1, Ter2 and Ter3; TO3TS1=BCD Ligne [26] with buses running between Boulogne-Calais-Dunkerque; TO1TS2 $=$ Lilas [27] with car sharing; TO2TS2 $=$ V'Lille [4.] with bike sharing; TO1TS3=carpooling. We consider a [6:00-10:00] a.m. window of a weekday. Weights $w \mathrm{k} \gamma$ are assigned so that the most important criterion has a 0.6 weight, 


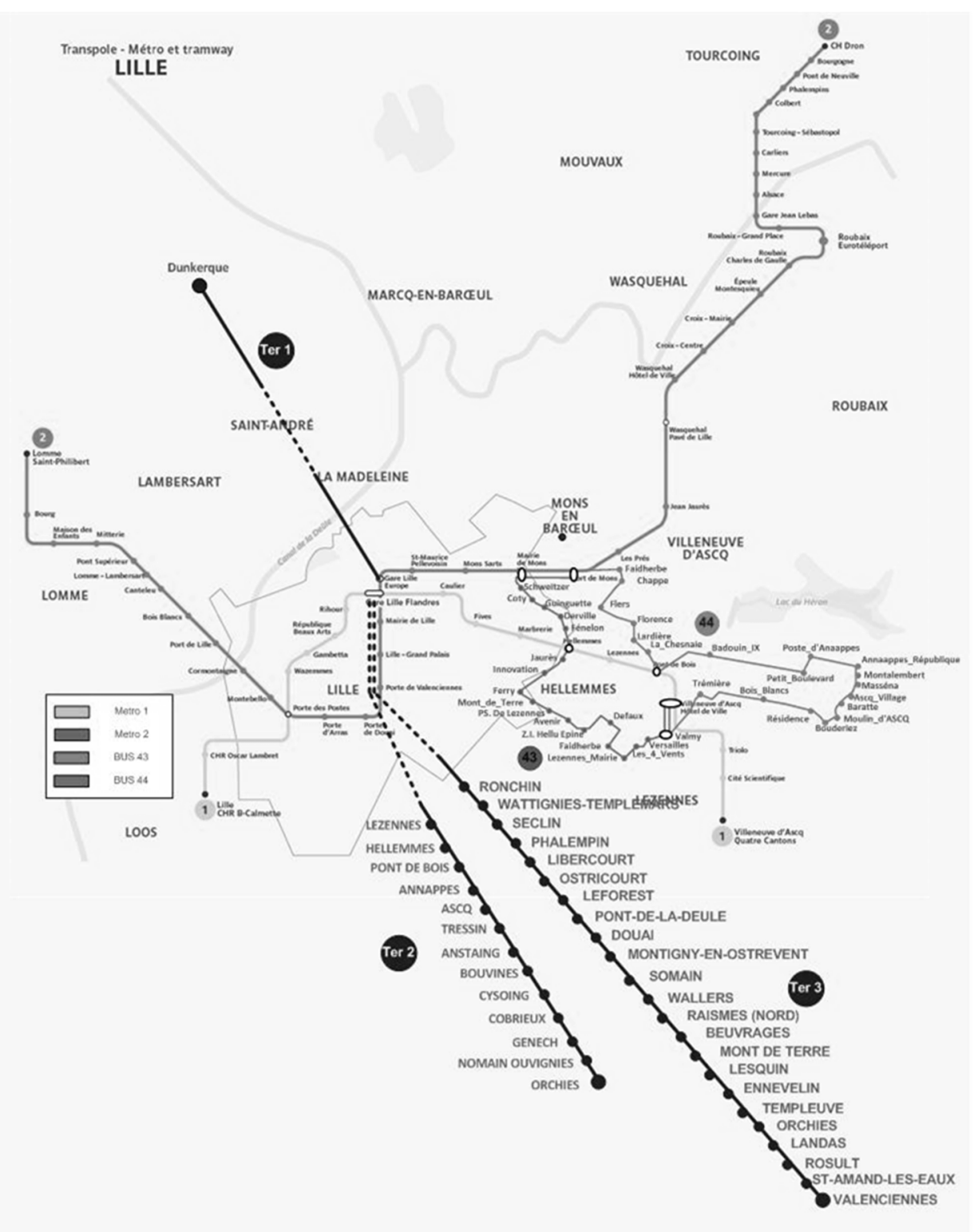

Fig. 12. The case study transportation network.

the second 0.3 , and the third 0.1 . We report average gas emissions [31], costs, and speeds of the transportation means in Table II. For private cars, extra-urban buses and urban buses, parameters regard the average emissions per vehicle: an average number of passengers respectively of 3,20, 20 is considered and calculate the average emission per person by the distance.

\section{A. Scenario 1}

The first scenario regards requests (extended from [12]):

$\mathrm{i}_{1}$ : Boulogne-Port de Lille [6:00-9:15];

$\mathrm{i}_{2}$ : Calais-Port de Lille [6:30-9:15];

$\mathrm{i}_{3}$ : Port de Lille-Pont de Bois [8:55-9:20];

$\mathrm{i}_{4}$ : Gambetta-Triolo [7:10-7:50].

Users' ordered preferences are: $\mathrm{i}_{1}, \mathrm{i}_{2}$ : extra-urban bus, subway, train, private car, $\mathrm{i}_{3}, \mathrm{i}_{4}$ : subway, private car. We consider a first optimization test with the ordered list: travel time, travel cost, gas emissions for all users. In the second optimization test we change the ordered preference list: gas emissions, travel cost, travel time.

Table III shows the ATIS results that are obtained in less than 3 minutes for both tests running on a Personal Computer and that we detail in the sequel.

The first optimization test results are (see Figs. 13 and 14):

$i_{1}:$.-1, Boulogne-Dunkerque, [6:15-7:35], extra-urban bus; 1-2, Dunkerque-Port de Lille, [8:..-8:5.], private car;

$\mathrm{i}_{2}$ : 3-1, Calais-Dunkerque, [6:50-7:35], extra-urban bus; 1-2, Dunkerque-Port de Lille, [8:00-8:50], private car;

$i_{3}$ : .-1, Port de Lille-Pont de Bois, [9:00-9:15], private car; $\mathrm{i}_{4}$ : 2-3, Gambetta-Triolo, [7:10-7:24], subway line 1.

Hence, since $i_{1}$ and $i_{2}$ share the same destination and have similar arrival times, the ATIS proposes them to increase 
TABLE II

TransPortation MEANS PARAMETERS

\begin{tabular}{llrl}
\hline $\begin{array}{l}\text { Transportation } \\
\text { means }\end{array}$ & $\begin{array}{l}\text { Average } \\
\text { emissions }\left[\mathbf{g C O}_{2} / \mathbf{k m}\right]\end{array}$ & $\begin{array}{l}\text { Average } \\
\text { costs }[\mathbf{\epsilon} / \mathbf{k m}]\end{array}$ & $\begin{array}{l}\text { Average } \\
\text { speeds }[\mathbf{k m} / \mathbf{h}]\end{array}$ \\
\hline Extra-urban bus & 13.74 & 0.18 & 57 \\
Train & 16.30 & 0.20 & 45 \\
Public car & 0 & 0.80 & 30 \\
Private car & 23.30 & 0.05 & 50 \\
Urban bus & 22.34 & 1.40 & 8 \\
Subway & 1.60 & 1.40 & 44 \\
Bike & 0 & 1.40 & 10 \\
\hline \hline
\end{tabular}

TABLE III

ATIS RESUlTS TO ITINERARY REQUESTS IN EXTRA-URBAN SCENARIO

\begin{tabular}{|c|c|c|c|c|c|c|}
\hline \multirow[t]{2}{*}{ Requests } & \multicolumn{3}{|c|}{ Test 1} & \multicolumn{3}{|c|}{ Test 2} \\
\hline & $\begin{array}{c}\text { Cost } \\
{[\epsilon]}\end{array}$ & $\begin{array}{c}\text { Time } \\
\text { ['] }\end{array}$ & $\begin{array}{c}\text { Gas } \\
\text { emission } \\
{\left[\mathrm{g} \mathrm{CO}_{2}\right]}\end{array}$ & $\begin{array}{c}\text { Cost } \\
{[€]}\end{array}$ & $\underset{\text { ['] }}{\text { Time }}$ & $\begin{array}{c}\text { Gas } \\
\text { emission } \\
{\left[\mathrm{g} \mathrm{CO}_{2}\right]}\end{array}$ \\
\hline Request $i_{1}$ & 15.76 & 155 & 2694 & 16.48 & 170 & 2329 \\
\hline Request $\mathrm{i}_{2}$ & 8.92 & 120 & 2227 & 9.64 & 135 & 1862 \\
\hline Request $\mathrm{i}_{3}$ & 0.62 & 15 & 326 & 0.62 & 15 & 326 \\
\hline Request $\mathrm{i}_{4}$ & 1.40 & 14 & 11 & 1.40 & 4 & 11 \\
\hline
\end{tabular}

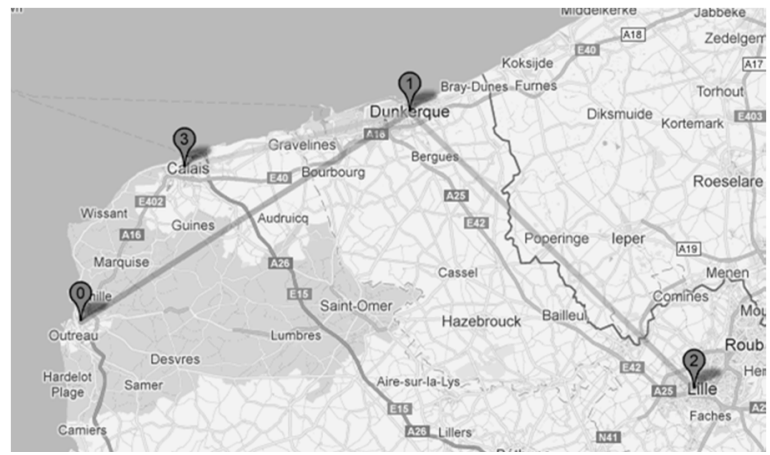

Fig. 13. The paths of $i_{1}(0-1-2)$ and $i_{2}(3-1-2)$ under test 1 , scenario 1 .

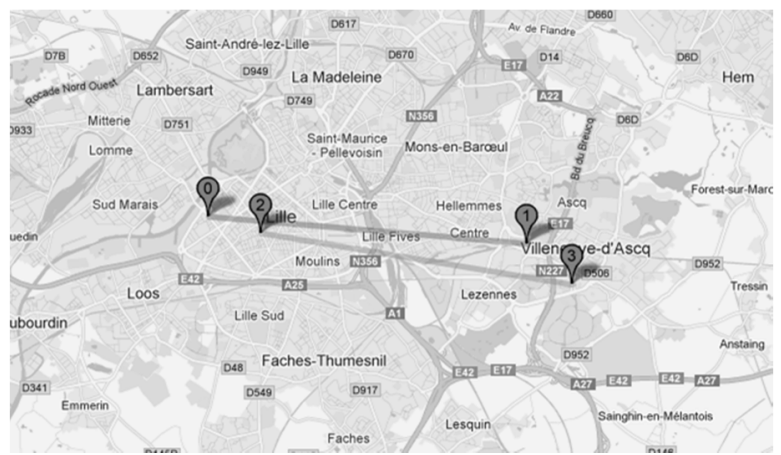

Fig. 14. The paths of $i_{3}(0-1)$ and $i_{4}(2-3)$ under tests 1 and 2 , scenario 1.

co-modality to reach by buses a common point and then share a private car (see Fig. 13). Moreover, the routes of $i_{3}$ and $i_{4}$ in the first optimization are independent (see Fig. 14).

The second test results are (see Figs. 14 and 15):

$i_{1}$ : 0-1, Boulogne-Dunkerque, [6:15-7:35], extra-urban bus; 1-2, Dunkerque-Gare Lille Europe, [8:21-8:55], train Ter1; 2-3, Gare Lille Europe-Port de Lille, [8:55-9:.5] subway line 2 ;

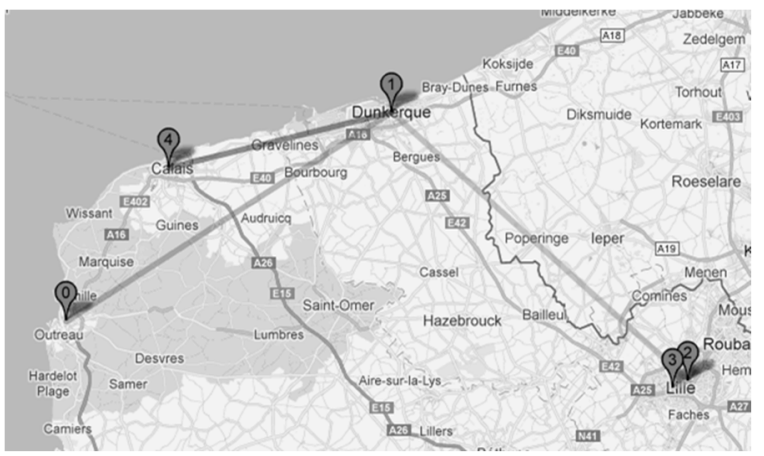

Fig. 15. The paths of $i_{1}(0-1-2-3)$ and $i_{2}(4-1-2-3)$ under test 2 , scenario 1 .

$\mathrm{i}_{2}$ : 4-1, Calais-Dunkerque, [6:50-7:30], extra-urban bus; 1-2, Dunkerque-Gare Lille Europe, [8:21-8:55], train Ter1; 2-3, Gare Lille Europe-Port de Lille, [8:55-9:05] subway line 2;

$i_{3}$ : 0-1, Port de Lille-Pont de Bois, [9:00-9:15], private car; $\mathrm{i}_{4}$ : 2-3, Gambetta-Triolo, [7:10-7:24], subway line 1.

Hence, in this case the ATIS proposes the first two users to use public transportation with three sub-routes with respect to the first optimization in which each path of the two users have two sub-routes shared with the other passenger (see Fig. 15). The last two users' routes are unchanged from the first test.

We enlighten the following issues. First, with the change of the criteria preferences, some solutions change: in the first test, minimization of travel time is more important than the environmental criterion; accordingly, the first and the second solutions share a private car; in the second test the most important criterion is emissions, hence, the ATIS suggests using the alternative solution resulting in a higher travel time: train and subway. Second, the change of solutions mainly regards multimodal paths that have more possibilities to adapt the itinerary to the user's preferences. Third, several solutions share at least one transportation means.

\section{B. Scenario 2}

The second scenario regards urban requests:

$\mathrm{i}_{5}$ : Port de Lille-Hellemmes [8:50-10:00];

$\mathrm{i}_{6}$ : Rihour-Fives [7:30-8:20];

i7: Cormontaigne-Gambetta [7:00-8:00];

i 8 : Pont de Bois-Mairie de Mons [7:00-9:00].

We consider the same criteria for each request in the order: travel time, travel cost, gas emissions. We execute two tests changing transportation means preference (the same for all users): for the first test, public services, namely, subway and urban buses, are selected. In the second test an interruption of public transport is considered, with bike and private cars only.

Table IV shows the results. The computational times are comparable to those obtained for the previous scenario.

The first optimization test results are (see Figs. 16 and 17):

i5: 0-1, Port de Lille-Gare Lille Flandres, [8:51-9:00], subway line 2; 1-2, Gare Lille Flandres-Hellemmes, [9:00-9:06], subway line 1;

$\mathrm{i}_{6}$ : 3-4, Rihour-Fives, [7:30-7:34], subway line 2;

i7: 0-1, Cormontaigne-Gare Lille Flandres, [7:04-7:12], subway line 2; 1-2, Gare Lille Flandres-Gambetta, [7:12-7:15], subway line 1 ; 
TABLE IV

ATIS RESULTS TO ITINERARY REQUESTS IN URBAN SCENARIO

\begin{tabular}{lcccccc}
\hline \hline Requests & \multicolumn{3}{c}{ Test 1 } & & Test 2 & \\
\hline & $\begin{array}{c}\text { Cost } \\
{[\boldsymbol{\epsilon}]}\end{array}$ & $\begin{array}{c}\text { Time } \\
{[']}\end{array}$ & $\begin{array}{c}\text { Gas } \\
\text { emission } \\
{\left[\mathbf{g ~ C O}_{2}\right]}\end{array}$ & $\begin{array}{c}\text { Cost } \\
{[€]}\end{array}$ & $\begin{array}{c}\text { Time } \\
{[']}\end{array}$ & $\begin{array}{c}\text { Gas } \\
\text { emission } \\
{\left[\mathbf{g ~ C O}_{2}\right]}\end{array}$ \\
Request $\mathbf{i}_{5}$ & 2.80 & 15 & 13 & 7.80 & 20 & 0 \\
Request $\mathbf{i}_{6}$ & 1.40 & 4 & 5 & 2.80 & 7 & 0 \\
Request $\mathbf{i}_{7}$ & 2.80 & 11 & 8 & 2.60 & 4 & 0 \\
Request $\mathbf{i}_{8}$ & 2.80 & 11 & 37 & 2.40 & 6 & 0 \\
\hline \hline
\end{tabular}

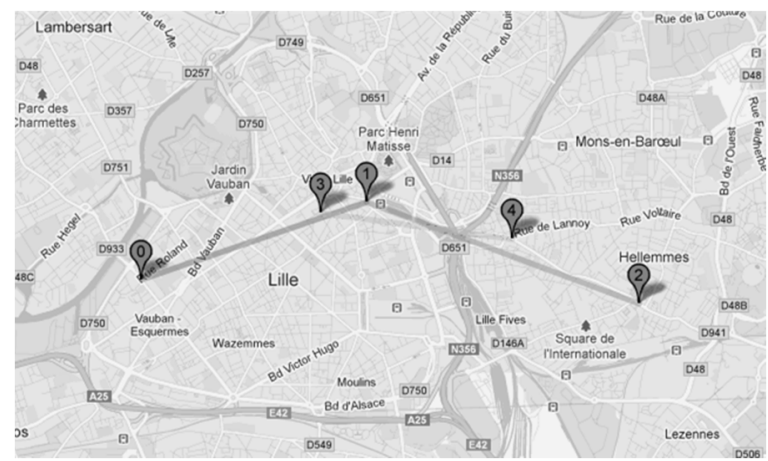

Fig. 16. The paths of $i_{5}(0-1-2)$ and $i_{6}(3-4)$ under test 1 , scenario 2 .

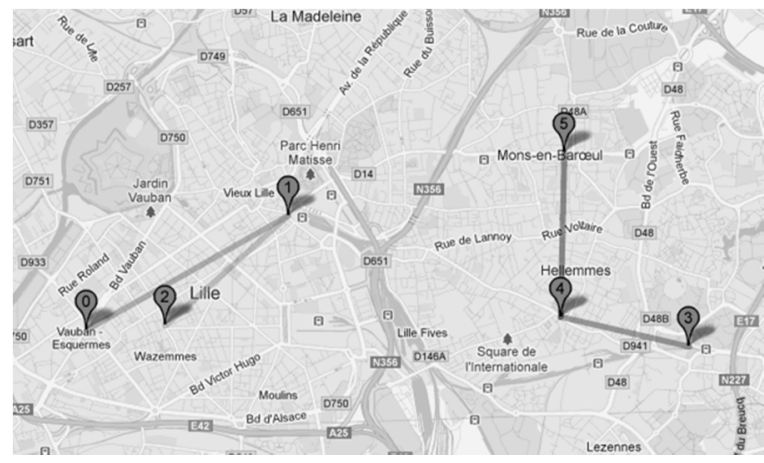

Fig. 17. The paths of $i_{7}(0-1-2)$ and is (3-4-5) under test 1 , scenario 2 .

$i_{8}$ : 3-4, Pont de Bois-Hellemes, [7:01-7:04], subway line 1; 4-5, Hellemmes-Mairie de Mons, [7:05-7:12], bus 44.

The second test results are (see Figs. 18 and 19):

i5: 0-1, Port de Lille-Cormontaigne, [8:00-8:54], bike; 1-2,

Cormontaigne-Hellemmes, [8:54-9:10], public car;

$\mathrm{i}_{6}$ : 3-4, Rihour-Fives, [7:3.-7:37], public car.

i7: 0-1, Cormontaigne-Wazemmes, [7:00-7:03], public car;

1-2, Wazemmes-Gambetta, [7:03-7:04], bike;

$i_{8}$ : 3-4, Pont de Bois-Mairie de Mons [7:00-7:06], public car.

Summing up, in the first test only the public transport component is used and the ATIS chooses subway and urban buses, providing a multimodal solution for the fourth request. In the second test, instead, public transport is avoided since it is not available and other means of transport answer demands.

\section{Discussion: Advantages and Limitations of the ATIS}

We now comment on the co-modality setting of our ATIS. We remark that the ATIS provides itineraries' suggestions to users based on their own (eventually different) preferences but

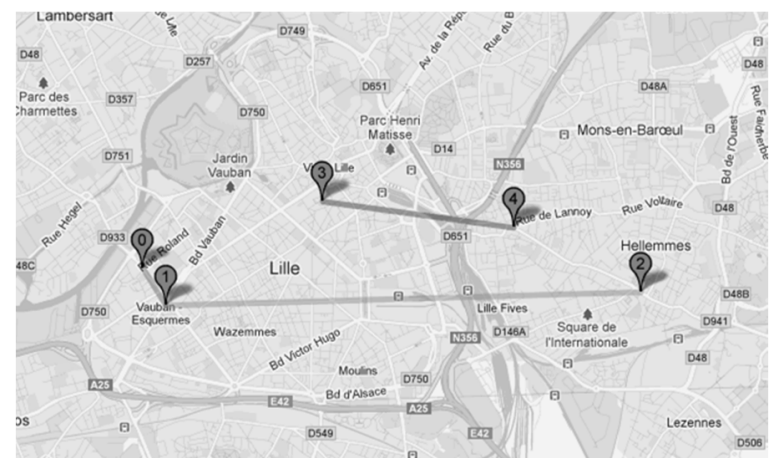

Fig. 18. The paths of $i_{5}(0-1-2)$ and $i_{6}(3-4)$ under test 2 , scenario 2 .

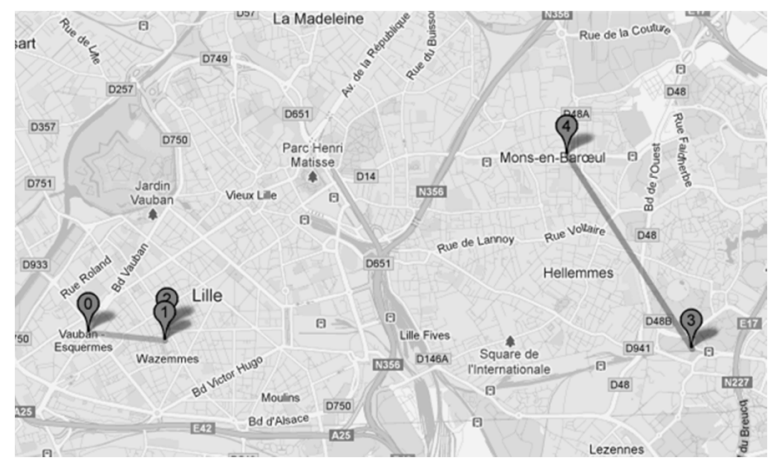

Fig. 19. The paths of i7 (0-1-2) and i8 (3-4-5) under test 2 , scenario 2 .

considering a system view, i.e., running an optimization on all the different requests collected in the considered instance. In fact, in principle some request may just consider cost or time minimization (thus, a selfish optimization) while making use of a co-modal optimization, allowing for instance the use of a private service such as car pooling together with the use of a public service such as for instance the subway, leads to a service penetration that single service use do not allow.

In addition, we remark that the current implementation of the ATIS is made on a Personal Computer, leading to results to the reported scenarios in less than 3 minutes. However, we remark that implementing the ATIS on a dedicated server prototype where the agents' decisions may be parallelized, deploying the distributed characteristics of multi-agent systems, these computational times may be consistently reduced. Preliminary experiments that we do not report for the sake of brevity have shown that computational times decrease to some tens of seconds.

Moreover, we comment on the ATIS scalability. To respond to simultaneous requests, the ATIS creates a coalition of agents. These agents, which are autonomous and intelligent, cooperate to respond as quickly as possible to requests. If further requests arrive and the created coalition has already finished the treatment of previous requests, it proceeds to handle these new requests. Otherwise, if the current coalition of agents is undergoing, a new coalition of agents is created to process new requests and so on. If an already formed coalition of agents is inactive for a specified time, then it is destroyed. If the ATIS does not receive any request or if all requests were already processed, then all agents' coalitions are destroyed and the ATIS system is reduced to a "watchdog" agent that waits 

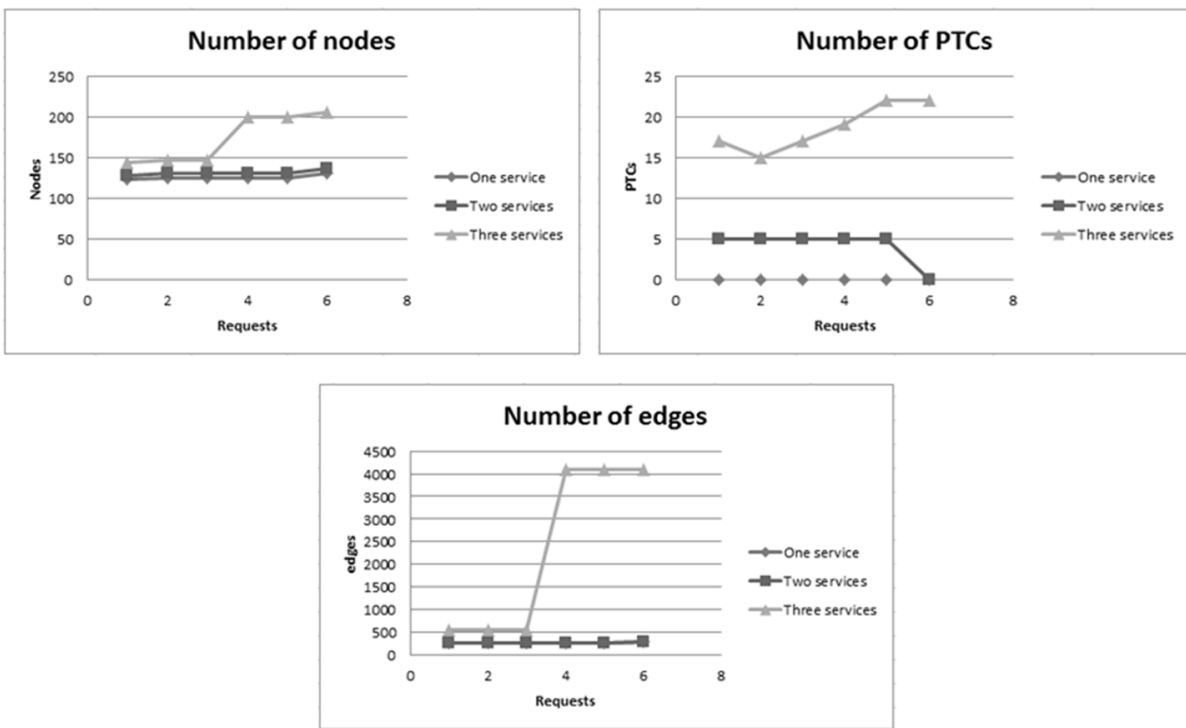

Fig. 20. Variation of the number of nodes, co-modal transfer points (PTCs) and edges with the number of requests and transportation services.

for the arrival of new requests. This concept ensures the vitality and scalability of the ATIS and allows handling a large number of requests without saturating the system, because the ATIS creates and destroys agents as needed.

In addition, to measure the impact on the ATIS of the number of requests and services, we make some further tests. We vary the requests and the number of services and we compare the variation of the number of nodes, co-modal transport points and edges, shown in Fig. 20. We remark from Fig. 20. that, in case of one transportation service, the number of co-modal transfer points is null. We also note that the number of edges increases in the case of including three transportation services. In addition, we remark some steadiness of the curves due to the similarity between the different requests. In fact, when the departure and arrival points are similar or geographically close, we obtain the same routes and so the same edges. Finally, we remark that there are some limits to the development and the use of the ATIS. Obstacles to overcome are economical and organizational. The ATIS must provide solutions taking into account the mobile telecommunication infrastructure and the independence of vehicles. In addition, the ATIS is based on a distributed architecture. So, transport operators have to share their databases in order to propose their services through ATIS.

However, nowadays, some operators are reticent and are not yet prepared to share their data within the same information system because of legal and political reasons. These limitations may prevent the massive use of an ATIS as consumer software. Moreover, the transport data updating in case of disturbances depends on the information providers' reactivity, in order to make available relevant and useful information to the ATIS. So, the data update can be considered as another limit of the ATIS because it assumes data are reliable.

VI. Conclusions AND PRospects

We propose a novel Advanced Traveler Information System (ATIS) for co-modal passengers' transportation based on a multi-agent system architecture to answer multi-criteria user requests. The multi-agent systems framework is selected due to its distributed feature that allows decomposing the trip planning problem into multiple simpler tasks. The presented ATIS can satisfy multiple requests with multiple conflicting criteria. We show that if a user changes its criteria preference, the itinerary may change and that this is accentuated for multimodal paths. Moreover, we show that the ATIS is able to propose solutions even when not all transportation means are available, for instance in case of strikes.

Numerous prospects exist for future research. They may regard using geographical coordinates to define the transportation network, adding some features to allow users change or personalize their path in real time, and minimizing the number of determined subroutes and hence of route combinations to speed up the ATIS. Moreover, including the proposed planning tool in a real time decision support system may be considered for devising an ATIS for real-time trip planning allowing for traffic guidance and reduction. In addition, we are working on implementing a reward system in our ATIS for incentivizing users choose more sustainable solutions. Further, we would like to integrate within the ATIS several alternative optimization algorithms from which the system may choose dynamically the best algorithm according to the transport problem complexity. Moreover, the ATIS may use responsive user interfaces in order to adapt the information representation, according to the user adopted device. Last but not least, we shall implement the ATIS on a dedicated server prototype to parallelize agents' decisions and reduce computational times, as already confirmed by some preliminary experiments.

\section{APPENDIX}

The following notation is used throughout the paper.

$i$ : transportation service index;

$j:$ transportation operator index;

$y$ : transportation means index;

$T S_{i}:$ transportation service;

$T O_{i j}$ : transportation operator for $i$-th service;

$T M_{i j y}$ : transportation means of $j$-th operator of $i$-th service;

$T S$ : set of $n$ available transportation services;

$T M$ : set of all transportation means; 
$c_{\gamma}:$ performance criterion;

$C$ : set of c criteria;

$\mathrm{C}_{\mathrm{k}} w_{k \gamma}$ : weight associated with $\gamma$-th criterion for $k$-th user;

$W$ : weight matrix;

$i_{k}=\left(d_{k}, a_{k}, W_{k}, V_{k}, C_{k}\right):$ itinerary request;

$k$ : user index;

$I$ : set of $K$ itinerary requests;

$d_{k}$ : departure point;

$a_{k}:$ arrival point;

$W_{k}=\left(t_{d k}, t_{a k}\right):$ time window;

$t_{d k}$ : minimum allowed departure time by user $k$ from $d_{k}$;

$t_{a k}$ : maximum allowed arrival time by user $k$ to $a_{k}$;

$V_{k}$ : list of transportation means allowed by user $k$;

$C_{k}$ : criteria ordered list for user $k$;

$S_{k}$ : route solution path of user $k$;

$S$ : set of route solution paths to $K$ requests;

$R C_{k}$ : set of all the possible routes answering request $k$;

$P_{k}$ : number of all the possible routes answering request $k$;

$R C_{k, p}$ subroutes combination that can serve the $k$-th request;

$R_{g}=\left(d_{g}, a_{g}, W_{g}, u_{g}, V_{g}\right)$ : subroute;

$g$ : subroute index;

$D\left(d_{g}, a_{g}\right)$ distance between starting and end points of subroute;

$T\left(d_{g}, a_{g}\right)$ travel time of subroute;

$X$ : number of all possible subroutes concerned by all requests;

$\Lambda$ : set of ordered couples with lists of users and vehicles sharing the same departure point and arrival point and allowing the same transportation operator;

$u_{g}$ : list of users that can use a subroute $g$;

$V_{g}$ : list of vehicles that can provide the trip for subroute $R_{g}$

$V_{h, R_{g}} h$-th vehicle of $V_{g}$ serving route $R_{g}$;

$V_{m}\left(V_{h, R_{g}}\right)$ average speed of vehicle $V_{h, R_{g}}$ travelling along route $R_{g}$;

$C_{k m}$ cost of a one Kilometer route;

$F_{e}\left(V_{h, R_{g}}\right)$ vehicle emission factor per Km;

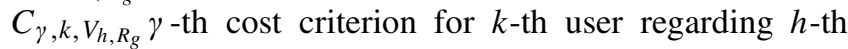
vehicle of $V_{g}$ serving subroute $R_{\mathrm{g}}$;

fitness $_{k}$ : fitness of $k$-th user;

$\mathrm{CH}(\mathrm{k}, \mathrm{h})$ : chromosome assignment for $k$-th user and $h$-th vehicle;

$\mathrm{CH}$ : chromosome matrix;

$G_{\mathbf{A}}=\left(N_{\mathbf{A}}, E_{\mathbf{A}}\right)$ : adjacency graph;

$\mathrm{G}(\mathrm{N}, \mathrm{E}) N_{\mathrm{A}}$ : set of nodes of adjacency graph ;

$E_{\mathrm{A}}$ : set of edges of adjacency graph;

$G_{\mathbf{C}}=(N, E, T S)$ : co-modal graph;

$N$ set of vertices of co-modal graph;

$E$ set of edges co-modal graph;

$e_{l}=\left(\left(n_{\mathrm{p}}, n_{\mathrm{q}}\right), T S_{i}\right):$ edge of $E$;

$D e_{1}$ : weight of edge $e_{l}$;

$G_{\mathbf{T}}=(C C, T R)$ transfer graph;

$C C_{\mathbf{i}}$ : component of transfer graph;

$C C$ : set of uni-service components;

$T R$ : set of virtual edges which interconnect components;

$P T C_{\mathrm{i}}$ : co-modal transfer point;

$R C *_{\mathrm{i}, \mathrm{d}_{\mathrm{k}}, \mathrm{a}_{\mathrm{k}}}$ : shortest path from $d_{k}$ to $a_{k}$ in $C C_{\mathrm{i}}$;

$R C *_{\mathrm{i}, \mathrm{d}_{\mathrm{k}}, \mathrm{PTC}_{\mathrm{i}}}$ : shortest path from $d_{k}$ to $P T C_{\mathbf{i}}$ in $C C_{\mathbf{i}}$;

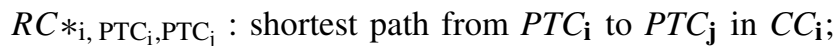
$R C *_{\mathrm{i}, \mathrm{PTC}_{\mathrm{j}}, \mathrm{a}_{\mathrm{k}}}$ : shortest path from $P T C_{\mathbf{j}}$ to $a_{k}$ in $C C_{\mathbf{i}}$.

$G_{\mathbf{S P T}}=\left(N_{\mathbf{S P T}}, E_{\mathbf{S P T}}\right)$ : shortest path transfer graph.

\section{REFERENCES}

[1] M. A. Abdel-Aty and M. F. Abdalla, "Examination of multiple mode/route-choice paradigms under ATIS," IEEE Trans. Intell. Transp. Syst., vol. 7, no. 3, pp. 332-348, Sep. 2006.

[2] J. L. Adler, G. Satapathy, V. Manikonda, B. Bowles, and V. J. Blue, "A multi-agent approach to cooperative traffic management and route guidance," Transp. Res. B, Methodol., vol. 39, no. 4, pp. 297-318, 2005.

[3] T. A. Arentze, "Adaptive personalized travel information systems: A Bayesian method to learn users' personal preferences in multimodal transport networks," IEEE Trans. Intell. Transp. Syst., vol. 14, no. 4 pp. 1957-1966, Dec. 2013.

[4] H. Ayed, C. Galvez-Fernandez, Z. Habbas, and D. Khadraoui, "Solving time-dependent multimodal transport problems using a transfer graph model," Comput. Ind. Eng., vol. 61, no. 2, pp. 391-401, 2011.

[5] F. Bellifemine, G. Caire, T. Trucco, and G. Rimassa. JADE Programmer's Guide, accessed on Oct. 14, 2015. [Online]. Available: http://jade.tilab.com/doc/programmersguide.pdf

[6] N. Borole, D. Rout, N. Goel, P. Vedagiri, and T. V. Mathew, "Multimodal public transit trip planner with real-time transit data," Procedias-Soc. Behavioral Sci., vol. 104, pp. 775-784, Dec. 2013.

[7] B. Chen and H. H. Cheng, "A review of the applications of agent technology in traffic and transportation systems," IEEE Trans. Intell. Transp. Syst., vol. 11, no. 2, pp. 485-497, Jun. 2010.

[8] C. Chen, D. Zhang, B. Guo, X. Ma, G. Pan, and Z. Wu, "TripPlanner: Personalized trip planning leveraging heterogeneous crowdsourced digital footprints," IEEE Trans. Intell. Transp. Syst., vol. 16, no. 3, pp. 1259-1273, Jun. 2015.

[9] G. Chen, S. Wu, J. Zhou, and A. K. H. Tung, "Automatic itinerary planning for traveling services," IEEE Trans. Knowl. Data Eng., vol. 26, no. 3, pp. 514-527, Mar. 2014.

[10] D. K. W. Chiu, O. K. F. Lee, H.-F. Leung, E. W. K. Au, and M. C. W. Wong, "A multi-modal agent based mobile route advisory system for public transport network," in Proc. 38th Annu. Hawaii Int. Conf. Syst. Sci., Jan. 2005, p. 92b.

[11] M. Dotoli, N. Epicoco, M. Falagario, and G. Cavone, "A timed Petri nets model for performance evaluation of intermodal freight transport terminals," IEEE Trans. Autom. Sci. Eng., vol. 13, no. 2, pp. 842-857, Apr. 2016

[12] M. Dotoli, S. Hammadi, K. Jeribi, C. Russo, and H. Zgaya, "A multi-agent decision support system for optimization of co-modal transportation route planning services," in Proc. IEEE CDC, Florence, Italy, Dec. 2013, pp. 911-916.

[13] H. M. Foo, H. W. Leong, Y. Lao, and H. C. Lau, "A multi-criteria, multi-modal passenger route advisory system," in Proc. IES-CTR, 1999, pp. $1-16$.

[14] T. Genin and S. Aknine, "Coalition formation strategies for selfinterested agents in task oriented domains," in Proc. IEEE/WIC/ACM Int. Conf. Web Intell. Intell. Agent Technol., Aug. 2010, pp. 205-212.

[15] Google Developers. Google Maps, accessed on Oct. 14, 2015. [Online]. Available: https://developers.google.com/maps/

[16] G. Götzenbrucker and M. Köhl, "Advanced traveller information systems for intelligent future mobility: The case of 'Anachb' in Vienna," IET Intell. Transp. Syst., vol. 6, no. 4, pp. 494-501, Dec. 2012.

[17] M. Houda, M. Khemaja, K. Oliveira, and M. Abed, "A public transportation ontology to support user travel planning," in Proc. IEEE 4th Int. Conf. Res. Challenges Inf. Sci., Nice, France, May 2010, pp. 127-136.

[18] E. Hyytiã, A. Penttinen, and R. Sulonen, "Non-myopic vehicle and route selection in dynamic DARP with travel time and workload objectives," Comput. Oper. Res., vol. 39, no. 12, pp. 3021-3030, 2012.

[19] J. Jariyasunant, D. B. Work, B. Kerkez, R. Sengupta, S. Glaser, and A. Bayen, "Mobile transit trip planning with real time data," in Transp. Res. Board Annu. Meeting, Washington, DC, USA, 2010, pp. $1-17$.

[20] K. Jeribi, H. Mejiri, H. Zgaya, and S. Hammadi, "Vehicle sharing services optimization based on multi-agent approach," in Proc. 18th IFAC World Congr., Jan. 2011, pp. 13040-13045. 
[21] K. Jeribi, H. Mejri, H. Zgaya, and S. Hammadi, "Distributed graphs for solving co-modal transport problems," in Proc. IEEE 14th Int. Conf. Intell. Transp. Syst., Oct. 2011, pp. 1150-1155.

[22] K. Jeribi, H. Zgaya, N. Zoghlami, and S. Hammadi, "Distributed architecture for a co-modal transport system," in Proc. IEEE Int. Conf. Sys., Man, Cybern., Anchorage, AK, USA, Oct. 2011, pp. 2797-2802.

[23] P. Kumar, V. Singh, and D. Reddy, "Advanced traveler information system for Hyderabad city," IEEE Trans. Intell. Transp. Syst., vol. 6 , no. 1, pp. 26-37, Mar. 2005.

[24] Q. Li and C. E. Kurt, "GIS-based itinerary planning system for multimodal and fixed-route transit network," in Proc. MID-Continent Transp. Symp., 2000, pp. 47-50.

[25] L. Li, H. Zhang, X. Wang, W. Lu, and Z. Mu, "Urban transit coordination using an artificial transportation system," IEEE Trans. Intell. Transp. Syst., vol. 12, no. 2, pp. 374-383, Jun. 2011.

[26] Ligne $B C D$, accessed on Oct. 14, 2015. [Online]. Available: http://www.ligne-bcd.com/

[27] Lilas, accessed on Oct. 14, 2015. [Online]. Available: http://www.lilasautopartage.com/

[28] Z. Michalewicz and D. B. Fogel, How to Solve It: Modern Heuristics. Berlin, Germany: Springer-Verlag, 2004.

[29] A. Nuzzolo, U. Crisalli, A. Comi, and L. Rosati, "Individual behavioural models for personal transit pre-trip planners," Transp. Res. Procedia, vol. 5, pp. 30-43, Dec. 2015.

[30] K. Park, M. G. H. Bell, L. Kaparias, and K. Bogenberger, "Adaptive route choice model for intelligent route guidance using a rulebased approach," Transp. Res. Rec., J. Transp. Res. Board, vol. 2000, pp. 88-97, Nov. 2007.

[31] RATP, accessed on Oct. 14, 2015. [Online]. Available: http://www.ratp.fr/

[32] K. Rehrl, S. Bruntsch, and H. J. Mentz, "Assisting multimodal travelers: Design and prototypical implementation of a personal travel companion," IEEE Trans. Intell. Transp. Syst., vol. 8, no. 1, pp. 31-42, Mar. 2007.

[33] L. Rothkrantz, D. Datcu, and M. Beelen, "Personal intelligent travel assistant a distributed approach," in Proc. Int. Conf. Artif. Intell., 2005, pp. $1-7$.

[34] S. Russell and P. Norvig, Artificial Intelligence: A Modern Approach. Upper Saddle River, NJ, USA: Pearson Education, 2009.

[35] S. Satunin and E. Babkin, "A multi-agent approach to intelligent transportation systems modeling with combinatorial auctions," Expert Syst. Appl., vol. 41, no. 15, pp. 6622-6633, 2014.

[36] SNCF, accessed on Oct. 14, 2015. [Online]. Available: http://www.sncf.com/en/passengers

[37] J.-M. Su, C.-H. Chang, and W.-C. Ho, "Development of trip planning systems on public transit in Taiwan," in Proc. IEEE Int. Conf. Netw., Sens. Control, Hainan, China, Apr. 2008, pp. 791-795.

[38] Transpole, accessed on Oct. 14, 2015. [Online]. Available: http://www.transpole.fr/

[39] G. Tumas and F. Ricci, "Personalized mobile city transport advisory system," in Information and Communication Technologies in Tourism. Vienna, Austria: Springer, 2009, pp. 173-184.

[40] V'Lille, accessed on Oct. 14, 2015. [Online]. Available: http://www.vlille.fr/

[41] J. Yang and Z. Luo, "Coalition formation mechanism in multi-agent systems based on genetic algorithms," J. Appl. Soft Comput., vol. 7, no. 2, 2007, pp. 561-568.

[42] M. Yin and M. Griss, "SCATEAgent: Context-aware software agents for multi-modal travel," in Proc. Appl. Agent Technol. Traffic Transp., 2005, pp. 69-84.

[43] J. Wang and T. Kaempke, "Shortest route computation in distributed systems," Comput. Oper. Res., vol. 31, no. 10, pp. 1621-1633, 2004.

[44] F. Y. Wang, P. B. Mirchandani, and S. Tang, "Guest editorial advanced traveler information systems and vision-based techniques for ITS," IEEE Trans. Intell. Transp. Syst., vol. 6, no. 1, pp. 1-4, Mar. 2005.

[45] J. W. Zhang, F. X. Liao, T. Arentze, and H. Timmermans, "A multimodal transport network model for advanced traveler information system," Proc.-Social Behavioral Sci., vol. 20, pp. 313-322, 2011.

[46] K. G. Zografos and K. N. Androutsopoulos, "Algorithms for itinerary planning in multimodal transportation networks," IEEE Trans. Intell. Transp. Syst., vol. 9, no. 1, pp. 175-184, Mar. 2008.

[47] M. Zolfpour-Arokhlo, A. Selemat, and S. Z. M. Hashim, "Route planning model of multi-agent system for a supply chain management," Expert Syst. Appl., vol. 40, no. 5, pp. 1505-1518, 2013.

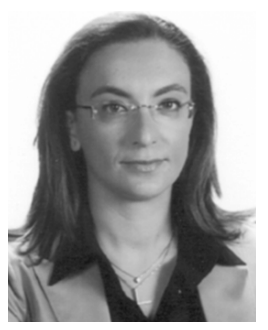

Mariagrazia Dotoli (M'96-SM'12) received the Laurea degree (Hons.) in electronic engineering and the Ph.D. degree in electrical engineering from the Politecnico di Bari, Italy, in 1995 and 1999, respectively.

She has been a Visiting Scholar with Paris 6 University and Technical University of Denmark. She is currently an Associate Professor in automatic control with the Department of Electrical and Electronic Engineering, Politecnico di Bari, where she joined as an Assistant Professor in 1999. In 2014, she received the Italian National Habilitation to Full Professor of Automatic Control. She has been the Vice Rector for research with the Politecnico di Bari and an elected member of its Academic Senate. Her research interests include modeling, identification, management, control and diagnosis of discrete event systems, Petri nets, manufacturing systems, logistics systems, and traffic networks.

Dr. Dotoli has authored over 180 peer-reviewed scientific papers. She has been a member of the International Program Committee of over 50 international conferences. She is the Program Co-Chair of the 2017 IEEE Conference on Automation Science and Engineering. She was also the Workshop and Tutorial Chair of the 2015 IEEE Conference on Automation Science and Engineering, the Special Session Co-Chair of the 2013 IEEE Conference on Emerging Technology and Factory Automation and the Chair of the National Committee of the 2009 IFAC Workshop on Dependable Control of Discrete Systems. She was a Co-Chairman of the Training and Education Committee of ERUDIT, the European Commission network of excellence for fuzzy logic and uncertainty modeling in information technology, and a key node representative of EUNITE, the European Network of excellence on Intelligent Technologies. She is the Editor-in-Chief of the IEEE Systems Man and Cybernetics Society e-Newsletter, and an Associate Editor of the IEEE Transactions on AUtomation SCIENCE AND ENGINEERING, the IEEE ROBOTICS AND AUTOMATION LETTERS, and the IEEE TRANSACTIONS ON CONTROL SYSTEMS TECHNOLOGY.

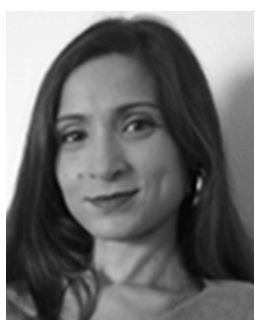

Hayfa Zgaya received the master's degree from Nantes University, France, in 2002, the Ph.D. degree from the Engineering French High School, Ecole Centrale de Lille, in 2007, and the highest academic qualification from University Lille 1, France, in 2014, allowing her to supervise research. She is currently an Associate Professor of Computer Science with the University of Lille 2, France. Her main research interests are in optimization, artificial intelligence, and logistics.

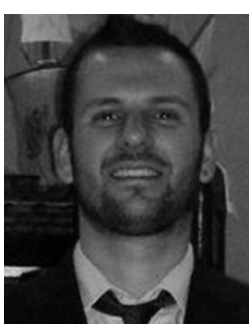

Carmine Russo received the Laurea degree (Hons.) in control engineering from the Politecnico di Bari, Bari, Italy, in 2012. He has been a Visiting Researcher with the Ecole Centrale de Lille, France, for a stage on multiagent systems for comodal transportation. $\mathrm{He}$ is currently a Consultant for Magneti Marelli-Powertrain Research and Development, where he is involved in the software design for automated manual transmission. His research interests include optimization, multiagent systems, and transportation networks.

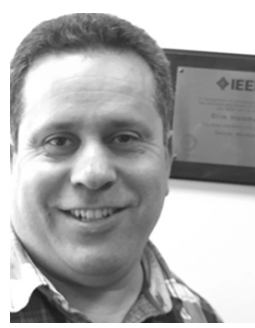

Slim Hammadi (SM'01) is currently a Full Professor of Modelization, Optimization, and Control of Complex Systems with the Ecole Centrale de Lille (French Grande Ecole). He is also a Director of the OSL Team, CNRS LAGIS Laboratory. He is Senior Member of the SMC and has served as a Referee for numerous journals, including the IEEE TRANSACTIONS ON SMC.

Mr. Hammadi was the President of the International Congress on Logistic and Transport LT'2004, MHOSI'2005, LT'2006, LT'2007, and LT'2009. He has authored 220 peer-reviewed scientific papers. His teaching and research interests focus on the areas of production control, production planning, computer science, discrete and dynamic programming, and computer integrated manufacturing. 